\title{
Phytogenic Feed Additives in Poultry: Achievements, Prospective and Challenges
}

\author{
Nedra Abdelli *, David Solà-Oriol (1) and José Francisco Pérez \\ Animal Nutrition and Welfare Service (SNIBA), Department of Animal and Food Science, Facultat de Veterinària, \\ Universitat Autònoma de Barcelona, 08193 Bellaterra, Spain; David.Sola@uab.cat (D.S.-O.); \\ josefrancisco.perez@uab.cat (J.F.P.) \\ * Correspondence: nedra.abdelli@uab.cat; Tel.: +34-93-581-1504
}

check for updates

Citation: Abdelli, N.; Solà-Oriol, D.; Pérez, J.F. Phytogenic Feed Additives in Poultry: Achievements, Prospective and Challenges. Animals 2021, 11, 3471. https://doi.org/ $10.3390 /$ ani11123471

Academic Editors: Luciana Rossi and Carlotta Giromini

Received: 4 November 2021

Accepted: 30 November 2021

Published: 6 December 2021

Publisher's Note: MDPI stays neutral with regard to jurisdictional claims in published maps and institutional affiliations.

Copyright: (c) 2021 by the authors. Licensee MDPI, Basel, Switzerland. This article is an open access article distributed under the terms and conditions of the Creative Commons Attribution (CC BY) license (https:/ / creativecommons.org/licenses/by/ $4.0 /)$.
Simple Summary: Plant secondary metabolites and essential oils also known as phytogenics are biologically active compounds that have recently attracted increased interest as feed additives in poultry production, due to their ability to promote feed efficiency by enhancing the production of digestive secretions and nutrient absorption, reduce pathogenic load in the gut, exert antioxidant properties and decrease the microbial burden on the animal's immune status. However, the mechanisms are far from being fully elucidated. Better understanding the interaction of phytogenics with gastrointestinal function and health as well as other feed ingredients/additives is crucial to design potentially cost-effective blends.

Abstract: Phytogenic feed additives have been largely tested in poultry production with the aim to identify their effects on the gastrointestinal function and health, and their implications on the birds' systemic health and welfare, the production efficiency of flocks, food safety, and environmental impact. These feed additives originating from plants, and consisting of herbs, spices, fruit, and other plant parts, include many different bioactive ingredients. Reviewing published documents about the supplementation of phytogenic feed additives reveals contradictory results regarding their effectiveness in poultry production. This indicates that more effort is still needed to determine the appropriate inclusion levels and fully elucidate their mode of actions. In this frame, this review aimed to sum up the current trends in the use of phytogenic feed additives in poultry with a special focus on their interaction with gut ecosystem, gut function, in vivo oxidative status and immune system as well as other feed additives, especially organic acids.

Keywords: phytogenics; performance; digestibility; microbiota; immunity; oxidant status; organic acids; microencapsulation; poultry

\section{Introduction}

Poultry production is undergoing a continuous challenge to develop management strategies to optimize chickens' efficiency while limiting food safety concerns. Traditionally, antimicrobials have been widely used for improving health and growth performance in poultry; however, the increased public awareness about the risk of developing crossresistance of pathogens to antibiotics has resulted in the gradual removal of antibiotics for therapeutic and prophylactic uses in food animals [1]. The shift away from antibiotic supplementation has resulted in a tremendous growth in research focusing on the implementation of effective alternative control methods, management and dietary amendments aiming to improve animal health, welfare, and productivity. A wide range of feed additives including a broad spectrum of essential oils and related compounds from botanical sources to organic acids [1,2], as well as probiotics and prebiotics [3], chemicals such as aldehydes [4], bacteriophages [5], zinc oxide [6], exogenous enzymes [7] and competitive exclusion products [8] have been used in animal production. Particularly, phytogenic feed additives 
(PFAs), also popularly referred as phytobiotics or botanicals, have gained an increasing interest as cost-effective feed additives with proven positive effects on broiler chickens' intestinal health. Indeed, antioxidative, immunomodulatory and growth-promoting effects have also been largely described in the literature. Therefore, the aim of the present review is to summarize the main results of some recent studies evaluating the effect of PFAs supplementation on the major components of bird gastrointestinal health and functionality, with special focus on nutrient digestibility, gut microbiota, immune system, oxidative status and growth performance of broilers and laying hens.

\section{Gastrointestinal Health and Functionality}

The regulation of gastrointestinal tract (GIT) function involves complex interactions among six major components, including the diet, effective digestion and absorption, normal and stable microbiota, effective immune status, gut mucosa, and neuroendocrine and motor function of the gut [9]. Both, diet composition (ingredients, nutrients and additives) and form, including structure and particle size, may affect the GIT functionality, especially through modulating the immune system and intestinal microbiota [9]. In fact, some dietary factors such as certain types of dietary fiber, trypsin inhibitors, phytate, lectins, undigested protein in the distal gastrointestinal tract, mycotoxins, as well as diets with poor nutrient balance, may affect the inflammatory process by modulating both pro-inflammatory and anti-inflammatory mechanisms [10] and thereby, disrupt the structural and functional integrity of the gut [11]. Conversely, feed additives such as phytobiotics, organic acids, enzymes, prebiotics, probiotics [12-14], functional foods and nutraceuticals [15] may play key roles in promoting overall health and growth performance.

The main attributes of an effective GIT functionality is an optimal digestion and absorption, maintenance of fluid and electrolyte balance, and elimination of waste products as well as maintenance of a barrier against antigens and pathogens [9]. The gastrointestinal compartments of healthy chickens are densely harbored by complex microbial communities which provide both nutrition and protection for the animal. Commensal microbiota may stimulate the development of immune system including the mucus layer, epithelial monolayer, the intestinal immune cells (e.g., cytotoxic and helper T-cells, immunoglobulin producing cells and phagocytic cells), and the lamina propia; thereby allowing to form a protective barrier between the host and the microbes [16]. Moreover, the microbiota of the distal gut (i.e., caeca and colon) uses the undigested feed to produce vitamins (e.g., vitamin K and vitamin B groups), amino acids, and short chain fatty acids (SCFAs: acetic acid, butyric acid and propionic acid) which eventually become available for the host. These SCFAs are considered of great interest for the host, for their bacteriostatic properties allowing to eliminate foodborne pathogens, such as Salmonella spp. [17], and as a source of energy which can stimulate gut epithelial cell proliferation and the gastrointestinal absorption surface [18].

On the other hand, impaired digestion and absorption results in a delivery of excess nutrients (such as starch, protein and fat) to the distal segments of the gastrointestinal tract, which induces alterations in the GIT microbial community resulting in qualitative and/or quantitative imbalance of normal microbiota in the small intestine. This imbalance is characterized by proliferation of pathogens which may lead to a sequential reaction in the GIT, including reduced intestinal barrier function (e.g., thinning of intestinal wall) and poor nutrient digestibility; and therefore, increasing the risk of bacterial translocation and inflammatory responses [19]. These negative effects on the symbiotic interactions between host and microbe lead to adverse effects on feed efficiency, productivity, and health of chickens [16].

The maintenance of GIT integrity is also crucial to ensure effective immune system as GIT is considered the largest organ of the immune system [20], that plays pivotal physiological role as barrier against antigens and pathogens. On the other hand, the GIT also possesses a neuroendocrine function through conveying neuroendocrine signals to the brain during digestion aiming to align the digestive and absorptive capacity of the GIT with 
the amount and composition of ingested food [11]. Therefore, it secretes gastrointestinal peptide hormones, such as gastrin, secretin and cholecystokinin in response to nutrient, neural or hormonal stimulation as well as by metabolic products of the gut microbiota. These peptide hormones are involved in the digestive and absorptive function of the GIT such as the regulation of gastric acid and pancreatic secretion, release of bile from the gall bladder and gut motor activity [9].

The diet is a main factor modulating the composition and the metabolic activity of the GIT microbiota [21]. In this regard, several feed additives have been developed focusing on enhancing immune response, reducing pathogen load in the GIT, promoting the colonization of the GIT with beneficial bacteria and stimulating digestion and absorption. The current review will discuss the effects of PFAs on the different components of GIT functionality.

\section{Phytogenics as an Alternative to Antimicrobials in Poultry Feeding}

A broad range of plants derived products may fall under the category of phytogenic feed additives. They may be classified either based on their origin (the part of the plant) into herbs (products from flowering, non-woody, and non-persistent plants from which leaves and flowers are used) and spices (non-leaf parts of plants, including seeds, fruits, bark or root with intensive taste or smell); or depending on the process used to derive the active ingredients as essential oils (EOs: volatile lipophilic substances obtained by cold extraction or by steam or alcohol distillation) and oleoresins (extracts derived by non-aqueous solvents). The bioactive components of PFAs are secondary metabolites being polyphenols the main group. Other bioactive compounds include terpenoids (monoterpenes, steroids...), phenolics (tannins), glycosides, and alkaloids [21]. The composition and concentration of these bioactive substances may vary according to several factors including the plant, parts of the plant, geographical origin, harvesting season, climatic conditions, processing techniques such as extraction, distillation and stabilization as well as storage conditions [22,23].

In recent years, PFAs have attracted an increasing attention as natural alternative to antibiotic growth promoters (AGPs) in poultry production which can be included in feeds as dried, solid, and ground form, or as extracts (crude, concentrated and purified) [24]. A wide variety of herbs and spices (thyme, oregano, cinnamon, rosemary, marjoram, yarrow, garlic, ginger, green tea, black cumin, coriander, among others) as well as EOs (from thymol, carvacrol, cinnamaldehyde, garlic, anise, rosemary, citruses, clove, ginger) have been used in poultry, individually or mixed, for their potential application as AGP alternatives [24]. Although the repertoire mechanisms of action of PFAs is not fully elucidated in poultry, one of their primary mode of action is related to their antimicrobial effects which allow controlling potential pathogens [25]. The results obtained in some recent studies will be reported with more details in the current review. However, some authors reported no positive effects of PFAs inclusion [26]. This discrepancy may be attributed to several factors, including the inherent variability of the botanic composition, as well the variability of the animal scenarios, environmental, management and sanitary conditions (i.e., including the likely presence of a pathogen challenge). The technique of treatment (cold, steam distillation, extraction or maceration with non-aqueous solvents...) has been also reported to change the active substances and related compounds in the final product [22].

Among PFAs, there is a rising interest in EOs for animal nutrition as some of these feed additives have been shown to possess a much higher biological activity compared to the raw material they were extracted from [27]. EOs are complex mixtures of volatile compounds, being mainly hydrocarbons (terpenes, sesquiterpenes), oxygenated compounds (alcohol, aldehydes, ketones) and a small percentage of non-volatile residues (paraffin, wax) [28]. Chemically, EOs consist fundamentally of two classes of compounds, the terpenes and phenylpropenes. Although, the effects of a mixture of EOs rely on the additive and synergy or antagonic effects of their components, 2 or 3 components may constitute up to $85 \%$ of the total mixture [29] and thus, contribute to its primary property [2]. In fact, thymol and 
carvacrol are the two main phenols which account for almost $80 \%$ of the EO of oregano and are the main contributors to its antibacterial and antioxidant activities. The compound pcymene is another dominant component of oregano EO [30]. Even though this component is not considered as an effective antimicrobial agent, it is a precursor for carvacrol which possesses higher preference for liposomal membranes, enabling carvacrol to be more easily transported into the cell [31].

EOs are perceived as growth promoters in poultry diets with strong antimicrobial and anticoccidial activities [2]. Thus, literature shows that growth promoting effects of EOs exist both abundantly and controversially, which makes it imperative to perform more in-depth research to understand the underlying mechanisms.

\section{Effects of Phytogenic Feed Additives on Chickens}

The effects of PFAs on the main metabolic and physiologic process like nutrient digestibility, intestinal microbiota, immunity, oxidant status and growth performance of broilers will be discussed with details below.

\subsection{Effects of Dietary PFA Supplementation on Growth Performance}

Several studies have been carried out using herbs, spices, and EOs and showed inconsistent results on chicken performance. Although some studies showed that PFAs have positive effects on body weight gain and FCR in chickens [32,33], others reported either an improved chicken body weight gain without affecting FCR $[34,35]$ or an enhanced feed conversion rate associated to a lack of effects on body weight or feed intake [36,37]. This inconsistency may be explained by several factors such as the botanical source, the concentration and the duration of supply of the active compounds, the feed composition, and the experimental challenging conditions, animal age and health status [38].

\subsubsection{Chickens Maintained under Non-Challenging Conditions}

Detailed results of some recent studies evaluating the effects of PFAs on growth performance of birds maintained under non-challenging conditions are presented in Tables 1-3. Dietary supplementation of EOs containing menthol, anethol and eugenol [39] as well as carvacrol alone [40], or combined with either thymol [41-43] or thymol and limonene [44] has been shown to promote growth performance of broiler chickens. Similar positive effects on production performance were obtained by supplementing laying hens diets with thymol and cinnamaldehyde [39], star anise oil [45], Citrullus lanatus EO [46], tea tree EO [47] and peppermint oil [48]. Several other studies have also evaluated the effects of supplementing black cumin (Nigella sativa L.) seeds on broilers, quails and laying hens. Although some authors failed to find any effect by supplementing Japanese quails diets with black cumin [49], others reported improved growth performance in quails [50,51] broiler chickens [52] and laying hens [53,54]. These growth-promoting effects have been attributed to the presence of a large number of pharmacologically active compounds such as thymoquinone, dithymoquinone, thymohydroquinone, nigellone, melanthin, nigilline, nigelamine, damascenone, $p$-cymene and pinene and a variety of essential nutrients including vitamins A, B, C, D and E, as well as minerals such as magnesium, calcium, phosphorus, potassium, iron, cobalt, zinc and manganese [41,55]. Moreover, curcuminoids and lipophilic turmeric extract containing curcumin and turmerones; known for their gastroprotective and anti-inflammatory activities, showed positive effects on growth performance of slow-growing [56] and fast-growing broiler chickens [57], respectively. Similar growth-promoting effects were observed by supplementing broiler chickens by a PFA of Aerva lanata, Piper betle, Cynodon dactylon, and Piper nigrum [58], Pulicaria gnaphalodes powder [59], Achyranthes japonica extract [60], Boswellia serrata [61] and bioactive olive pomace extract from Olea europaea [62] as well as laying hens by fennel seeds or red pepper [53] a mixture of Punica granatum, Thymus vulgaris, and Allium sativum [63] and dietary Nettle (Urtica cannabina) [64]. However, egg weight, laying rate and FCR were not improved by 
dietary supplementation of either EO of star anise (Illicium verum Hook.f.) [45] or a mixture of $13.5 \%$ thymol and $4.5 \%$ cinnamaldehyde [65].

\subsubsection{Chickens Maintained under Challenging Conditions}

A large number of studies have been performed to investigate the positive effects of PFAs on broiler chickens subjected to challenges related to environmental conditions including heat stress, lipopolysaccharide (LPS) and pathogens such as Clostridium perfringens, Eimeria spp, Salmonella typhimurium, or Escherichia coli, among others. Results of some recent studies are illustrated in Tables 2 and 4.

Heat stress in chickens induces a tight junction disruption that may lead to increased gut permeability and eventually to a dysregulation of the body's homeostasis [66], and thereby poor nutrient absorption, increased secretion of electrolytes and water in gastrointestinal tract leading to compromised performance [67]. However, the adverse effects caused by heat stress on broiler growth performance have been shown to be alleviated by the supplementation of turmeric rhizome powder [68], enzymatically treated Artemisia annua [69], ginger [70] and curcumin [71]. In ovo injection of black cumin (Nigella sativa) extract improved post-hatch performance of thermally challenged broiler chickens during incubation [72]. Similarly, 59-week-old cold-stressed laying hens supplemented with oregano EO showed improved FCR and egg production from week 9 to 12 of a 12-week feeding trial [73].

Numerous studies have been also performed to discern the efficacy of PFAs in chickens subjected to coccidiosis and necrotic enteritis (NE) classified among the most significant diseases affecting the poultry industry, which have become more prominent in the wake of policies to reduce the use of antibiotics in animal production. Clostridium perfringenschallenged broilers supplemented with PFAs consisting of either benzo [c]phenanthridine alkaloids from Macleaya cordata, active component of carvacrol from oregano $(4.95 \mathrm{~g} / 100 \mathrm{~g})$, cinnamaldehyde from cinnamon $(2.97 \mathrm{~g} / 100 \mathrm{~g})$, and capsaicin from paprika $(1.98 \mathrm{~g} / 100 \mathrm{~g})$, or EO of thyme and anise as leading active ingredients and other including oregano, carvacol, yucca extract and cinnamaldehyde exhibited similar growth performance as the control non-infected group [74]. Microcapsules with a blend of EOs (thyme, peppermint, savoury, and black pepper) at the dose of $0.5,1$, and $2 \mathrm{~kg} /$ ton in the $C$. perfringens-challenged broiler chickens results in raising final weight and total feed intake [75]. However, supplementation of EO containing $25 \%$ thymol and $25 \%$ carvacrol as active components, did not influence the growth performance during $\mathrm{d} 0$ to 14 and tended to linearly reduce the FCR between 14 and $28 \mathrm{~d}$ of age of Clostridium perfringens challenged broilers [76]. Regarding coccidiosis, the supplementation of a cashew nut shell oil and commercial castor oil blend allowed a recovery in performance similar to that observed with birds receiving the ionophore monensin during the accumulated experimental period (1 to $42 \mathrm{~d}$ ) [77]. Hussein et al. reported that combinations of peppermint, chamomile and prebiotic yeast cell wall were as effective as salinomycin in preventing the decline in the weight gain and FCR performance of coccidiosis-challenged broilers [78]. However, the effects of dietary $Y u c c a$-derived saponin supplementation on growth performance seem to be dependent on broiler health status. Although Su et al. [79] reported that saponin supplementation via an extract from $Y$. schidigera serves as an effective growth promoter in non-challenged broilers, Oelshlager et al. [80] found no significant influence of Yucca extract on growth responses of broilers during a mixed coccidian challenge. This suggests a reduction in the bioefficacy of saponins when used during an immune challenge [80].

Similarly, a 20-day experiment showed that dietary curcumin supplementation from day 12 to 20 failed to positively affect growth performance of broiler chickens challenged with Eimeria species on day 14 of age [81]. These authors attributed the lack of effect to the short period of supplementation and suggested that it might be beneficial if curcumin is fed in broilers for a 42-d period. This suggestion was based on the results of Rajput et al. [82], showing that birds fed curcumin-supplemented diets for $42 \mathrm{~d}$ exhibited significant increase 
in the BW and feed efficiency during the finisher stage (22-42 d), whereas no significant difference in growth performance was observed during the starter phase (0-21 d).

PFAs supplementation has been also shown to alleviate the effects on the broilers growth performance of other pathogens, such as Achyranthes bidentate under Escherichia coli challenge [83], resveratrol under Escherichia coli challenge [84], a PFA consisting of various nutritional acids and four different alkaloids obtained from special plants under Salmonella typhimurium challenge [85], and Allium hookeri roots in LPS-induced young broiler chickens.

Table 1. Effects of dietary supplementation of PFAs on growth performance of broilers under non-challenging conditions.

\begin{tabular}{|c|c|c|c|c|c|c|c|c|}
\hline \multirow[t]{2}{*}{ Feed Additive } & \multirow[t]{2}{*}{ Major Components } & \multirow{2}{*}{$\begin{array}{c}\text { Dose, } \\
\text { (mg/kg } \\
\text { Diet) }\end{array}$} & \multirow[t]{2}{*}{ Diet } & \multirow[t]{2}{*}{ Age } & \multicolumn{3}{|c|}{$\begin{array}{l}\text { Treatment Effects (\%, } \\
\text { Compared to Control) }\end{array}$} & \multirow[t]{2}{*}{ References } \\
\hline & & & & & BW & ADFI/FI & FCR & \\
\hline $\begin{array}{l}\text { Olea europaea } \\
\text { extract }\end{array}$ & Triterpenes ( $10 \%)$ polyphenols $(2 \%)$ & 750 & $\begin{array}{l}\text { Wheat- } \\
\text { soybean meal } \\
\text { based diet }\end{array}$ & $21-42 d$ & NM & NS & -7.9 & [62] \\
\hline $\begin{array}{l}\text { Achyranthes } \\
\text { japonica extract }\end{array}$ & $\begin{array}{l}\text { Flavonoid }(1.15 \mathrm{mg} / \mathrm{g}), \text { polyphenol } \\
(4.26 \mathrm{mg} / \mathrm{g}) \text { and saponin }(0.47 \mathrm{mg} / \mathrm{g})\end{array}$ & 1000 & $\begin{array}{l}\text { Corn-soybean } \\
\text { meal based } \\
\text { diet }\end{array}$ & $0-35 d$ & 3.5 & -2.4 & -6.2 & [60] \\
\hline EOs & Carvacrol (20\%) and thymol (25\%) & 200 & $\begin{array}{l}\text { Corn-soybean } \\
\text { meal based } \\
\text { diet }\end{array}$ & $29-42 d$ & NS & -9.6 & -11.8 & [43] \\
\hline EOs & $\begin{array}{c}\text { Carvacrol }(5 \%) \text {, cinnamaldehyde }(3 \%), \\
\text { and capsicum oleoresin }(2 \%)\end{array}$ & 100 & $\begin{array}{l}\text { Corn-soybean } \\
\text { or wheat } \\
\text {-soybean meal } \\
\text { based diet }\end{array}$ & & 16.4 & 6.1 & -9.4 & [86] \\
\hline $\begin{array}{l}\text { Aerva lanata, } \\
\text { Cynodon dactylon, } \\
\text { Piper nigrum and } \\
\text { Piper betle }\end{array}$ & $\begin{array}{c}\text { Phenolic acid contents } \\
(10,176.8 \mu \mathrm{g} / \mathrm{g}) \text {, flavonoids } \\
(53.0 \mu \mathrm{g} / \mathrm{g}), \text { other }(220.2 \mu \mathrm{g} / \mathrm{g})\end{array}$ & 10,000 & $\begin{array}{l}\text { Corn-soybean } \\
\text { meal based } \\
\text { diet }\end{array}$ & $0-42 \mathrm{~d}$ & 14.1 & NS & -14.0 & [58] \\
\hline $\begin{array}{l}\text { Pulicaria } \\
\text { gnaphalodes } \\
\text { powder }\end{array}$ & $\begin{array}{l}\text { Phenolic compounds, alkaloids, } \\
\text { terpenoids, and triterpene saponins }\end{array}$ & 3000 & $\begin{array}{l}\text { Corn-soybean } \\
\text { meal based } \\
\text { diet }\end{array}$ & $0-42 \mathrm{~d}$ & 4.3 & NS & -3.0 & [59] \\
\hline $\begin{array}{l}\text { Standardized } \\
\text { lipophilic } \\
\text { turmeric extract }\end{array}$ & $\begin{array}{l}3.1 \% \text { of curcuminoids content and } \\
\text { terpenes (turmerones) }\end{array}$ & 10,000 & NM & $0-42 \mathrm{~d}$ & 9.0 & 1.6 & -7.7 & [57] \\
\hline EOs & $\begin{array}{c}\text { Carvacrol }(63.5 \%), \text { thymol }(3.4 \%) \text { and } \\
\text { paracymene }(13.1 \%)\end{array}$ & $400 \mu \mathrm{L}$ & $\begin{array}{l}\text { Corn-wheat- } \\
\text { soybean meal } \\
\text { based diet }\end{array}$ & $28-43 d$ & 4.2 & NS & -3.9 & [40] \\
\hline Thyme powder & $\begin{array}{c}\text { Major EO (thymol (50.48\%), } \\
\gamma \text {-terpinene (11.03\%), P-cymene } \\
\text { (9.77\%), and carvacrol (4.30\%)), } \\
\text { phenolic acids (salicylic acid } \\
(2450.03 \text { ppm), ellagic acid } \\
(1240.42 \text { ppm)) and flavonoid } \\
\text { compounds }\end{array}$ & 5000 & $\begin{array}{l}\text { Corn-soybean } \\
\text { meal-based } \\
\text { diet }\end{array}$ & $0-42 \mathrm{~d}$ & 4.6 & 3.3 & NS & [87] \\
\hline $\begin{array}{l}\text { EOs (oregano, } \\
\text { anise, and citrus } \\
\text { peel; CBP) }\end{array}$ & $\begin{array}{l}\text { Carvacrol: } 102 \mathrm{~g} \text { of the chemical } \\
\text { component/kg of CBP }\end{array}$ & 150 & $\begin{array}{l}\text { Corn-soybean } \\
\text { meal-based } \\
\text { diet }\end{array}$ & $0-42 \mathrm{~d}$ & NS & -5.3 & NS & [88] \\
\hline $\begin{array}{l}\text { Combination of } \\
\text { herbs, spices, } \\
\text { EOs and extracts }\end{array}$ & $\begin{array}{c}\text { Mainly EOs from mint, star anise and } \\
\text { cloves }\end{array}$ & 100 & $\begin{array}{l}\text { Corn-soybean } \\
\text { meal-based } \\
\text { diet }\end{array}$ & $0-42 \mathrm{~d}$ & 7.0 & NS & NS & [39] \\
\hline $\begin{array}{l}\text { EO (powdered } \\
\text { and matrix- } \\
\text { encapsulated } \\
\text { form) }\end{array}$ & $\begin{array}{l}\text {-Powdered: menthol and anethole } \\
\text {-Encapsulaed: carvacrol, thymol, } \\
\text { and limonene }\end{array}$ & $\begin{array}{l}150 \\
100\end{array}$ & $\begin{array}{l}\text { Corn-wheat- } \\
\text { soybean meal } \\
\text { based diet }\end{array}$ & $0-42 \mathrm{~d}$ & $\begin{array}{l}\text { NS } \\
2.4\end{array}$ & $\begin{array}{l}\text { NS } \\
\text { NS }\end{array}$ & $\begin{array}{l}\text { NS } \\
\text { NS }\end{array}$ & [44] \\
\hline EOs & $\begin{array}{c}\text { Oregano containing carvacrol } \\
(26.4 \mathrm{mg} / \mathrm{kg}) \text { or thymol }(13 \mathrm{mg} / \mathrm{kg})\end{array}$ & $\begin{array}{l}300 \\
600\end{array}$ & $\begin{array}{l}\text { Corn-soybean } \\
\text { meal-based } \\
\text { diet }\end{array}$ & $0-42 \mathrm{~d}$ & $\begin{array}{l}7.8 \\
9.6\end{array}$ & $\begin{array}{l}4 \\
8\end{array}$ & $\begin{array}{l}\text { NS } \\
\text { NS }\end{array}$ & [41] \\
\hline $\begin{array}{l}\text { Spices: Nigella } \\
\text { sativa seeds }\end{array}$ & $\begin{array}{l}\text { Thymoquinone, dithymo- quinone, } \\
\text { thymohydroquinone, nigellone, } \\
\text { melanthin, nigilline, nigelamine, } \\
\text { damascenone, } p \text {-cymene and pinene }\end{array}$ & $\begin{array}{l}10,000 \\
20,000\end{array}$ & $\begin{array}{l}\text { Corn-soybean } \\
\text { meal-based } \\
\text { diet }\end{array}$ & $0-35 \mathrm{~d}$ & 3 & NS & 5.6 & [52] \\
\hline
\end{tabular}


Table 2. Effects of dietary supplementation of PFAs on growth performance of laying hens.

\begin{tabular}{|c|c|c|c|c|c|c|}
\hline Feed Additive & Major Components & $\begin{array}{l}\text { Dose (mg/kg } \\
\text { Diet) }\end{array}$ & Diet & Line and Age & Main Findings & References \\
\hline \multicolumn{7}{|c|}{ Non-Challenging Conditions } \\
\hline $\begin{array}{c}\text { Mentha arvensis } \\
\text { (MA) and Geranium } \\
\text { thunbergii (GT) } \\
\text { extracts }\end{array}$ & $\begin{array}{l}\text { MA: menthol, isomenthol, } \\
\text { neomenthol, p-cymene, } \\
\text { d-menthone, eugenol, and } \\
\text { cineol } \\
\text { GT: citronellol, } \\
\text { isomenthone, and } \\
\text { geraniin }\end{array}$ & $\begin{array}{l}100,500 \text { and } \\
1000\end{array}$ & $\begin{array}{l}\text { Corn-wheat- } \\
\text { soybean meal } \\
\text { based diet }\end{array}$ & $\begin{array}{l}\text { Hy-Line Brown } \\
\text { layers } \\
\text { (28-44 weeks) }\end{array}$ & $\begin{array}{l}\uparrow \mathrm{FI}, \text { egg } \\
\text { production and } \\
\text { egg weight }\end{array}$ & [89] \\
\hline $\begin{array}{l}\text { Fermented pine } \\
\text { (Pinus densiflora) } \\
\text { needle extract }\end{array}$ & $\begin{array}{l}\alpha \text {-pinene, caryophyllene, } \\
\text { beta-pinene and } \\
\text { bisbenzene, camphene, } \\
\text { borneol, phellandrene, } \\
\text { quercetin, kaempferol, } \\
\text { and terpene }\end{array}$ & 2.5 and 5 & $\begin{array}{l}\text { Corn-soybean } \\
\text { meal-based diet }\end{array}$ & $\begin{array}{l}\text { Hy-Line Brown } \\
\text { laying hens } \\
\text { (40-46 weeks) }\end{array}$ & $\begin{array}{l}\uparrow \mathrm{FI} \text {, egg } \\
\text { production and } \\
\text { egg mass }\end{array}$ & [90] \\
\hline $\begin{array}{c}\text { Fermented } \\
\text { Schisandra chinensis }\end{array}$ & - & & & & & \\
\hline $\begin{array}{l}\text { pomace (SC), } \\
\text { fermented Pinus } \\
\text { densiflora (PD) } \\
\text { needle extract, and } \\
\text { Allium tuberosum } \\
(\text { AT) powder }\end{array}$ & $\begin{array}{l}\text { PD: phenolics, flavonoids, } \\
\text { and tannins } \\
\text { AT: organosulfur } \\
\text { compounds, polyphenols, } \\
\text { and saponins }\end{array}$ & 1000 and 3000 & $\begin{array}{l}\text { Corn-soybean } \\
\text { meal-based diet }\end{array}$ & $\begin{array}{l}\text { Hy-line brown } \\
\text { laying hens } \\
\text { (48-54 weeks) }\end{array}$ & $\begin{array}{c}\text {-=Egg production, } \\
\text { daily egg mass } \\
\text { and FCR. } \\
-\uparrow \text { FI }\end{array}$ & [91] \\
\hline $\begin{array}{c}\text { Dry leaf extract of } \\
\text { peppermint (Mentha } \\
\text { piperita } L .)\end{array}$ & Menthol & $\begin{array}{l}0,74,148,222 \\
\quad \text { and } 296\end{array}$ & $\begin{array}{l}\text { Corn-soybean } \\
\text { meal-based diet }\end{array}$ & $\begin{array}{l}\text { Bovans Brown } \\
\text { laying hens } \\
\text { (32-44 weeks) }\end{array}$ & $\begin{array}{c}\uparrow \mathrm{FI}, \text { egg } \\
\text { production, egg } \\
\text { weight and egg } \\
\text { mass }\end{array}$ & [48] \\
\hline $\begin{array}{l}\text { Citrullus lanatus } \\
\text { EOs }\end{array}$ & $\begin{array}{c}\text { Phenolics }(1.57 \mathrm{mg} / 100 \mathrm{~g}) \\
\text { Sterols }(600.56 \mathrm{mg} / 100 \mathrm{~g}) \\
\text { Flavonoids }(163.5 \mathrm{mg} \\
\text { RE } / \mathrm{kg})\end{array}$ & 1000 and 2000 & $\begin{array}{l}\text { Corn-soybean } \\
\text { meal-based diet }\end{array}$ & $\begin{array}{l}\text { White Leghorn } \\
\text { laying hens } \\
\text { (18-26 weeks) }\end{array}$ & $\begin{array}{l}\uparrow \text { Weight gain, } \\
\text { ADFI, ADG and } \\
\text { egg mass; } \downarrow \text { FCR }\end{array}$ & [46] \\
\hline $\begin{array}{l}\text { Tea tree (Melaleuca } \\
\text { alternifolia) EO }\end{array}$ & $\begin{array}{c}\text { Terpinen-4-ol }(40.0 \%) \\
\gamma \text {-Terpinene }(23.0 \%) \text { and } \\
\alpha \text {-Terpinene }(10.4 \%)\end{array}$ & 40 and 80 & NM & $\begin{array}{l}\text { Lohmann } \\
\text { Brown hens } \\
\text { (55-58 weeks) }\end{array}$ & $\begin{array}{c}\uparrow \text { Daily egg } \\
\text { production and } \downarrow \\
\text { FCR }\end{array}$ & [47] \\
\hline $\begin{array}{l}\text { Thyme (Thymbra } \\
\text { spicata) and } \\
\text { Rosemary } \\
\text { (Rosemarinus } \\
\text { officinalis) }\end{array}$ & $\begin{array}{c}\text {-Thyme: Carvacrol } \\
(87.81 \%), \text { thymol }(9.58 \%), \\
\text { L-Linalool }(0.86 \%), \\
\text { borneol }(0.74 \%) \\
\text {-Rosemary: } 1.8 \text { cineole } \\
(34.08 \%), \text { camphor } \\
(27.95 \%), \text { alpha-Pinene } \\
(14.50 \%), \text { borneol } 1(8.65 \%), \\
\text { alpha-Terpineol }(7.39 \%), \\
\text { alpha-Thujone }(1.09 \%), \\
\text { camphene }(0.55 \%)\end{array}$ & $\begin{array}{l}1000 \text { for each } \\
\text { source }\end{array}$ & $\begin{array}{l}\text { Corn-soybean } \\
\text { meal-based diet }\end{array}$ & $\begin{array}{l}\text { Bovans-White } \\
\text { (48-56 weeks) }\end{array}$ & $\begin{array}{c}\text {-No effects on } \\
\text { FCR } \\
-\downarrow \text { Egg production } \\
\text { and egg weight }\end{array}$ & [92] \\
\hline $\begin{array}{l}\text { Cumin (Cuminum } \\
\text { cyminum L.) seed oil }\end{array}$ & $\begin{array}{c}\text { Cuminol, cuminique } \\
\text { alcohol, cuminaldehyde, } \\
\text { cymine, phellandrene, } \\
\text { carvone, cymol, terpenes, } \\
\alpha \text {-pinene ... }\end{array}$ & 500 & $\begin{array}{l}\text { Corn-soybean } \\
\text { meal-based diet }\end{array}$ & $\begin{array}{l}\text { Boven hens } \\
\text { (24-30 weeks) }\end{array}$ & $\begin{array}{c}=\text { Egg production } \\
\text { rate, egg mass } \\
\text { and FI } \\
\downarrow \text { FCR and } \uparrow \text { egg } \\
\text { weight }\end{array}$ & [93] \\
\hline Eucalyptus leaves & Polyphenols & $\begin{array}{l}500,800 \text { and } \\
1200\end{array}$ & $\begin{array}{l}\text { Corn-soybean } \\
\text { meal-based diet }\end{array}$ & $\begin{array}{l}\text { Yueqinhuang } \\
\text { laying hens } \\
\text { (35-44 weeks) }\end{array}$ & $\begin{array}{l}\uparrow \text { Egg production } \\
\text { and egg mass }\end{array}$ & [94] \\
\hline $\begin{array}{c}\text { Fennel seeds }(F), \\
\text { black cumin }(B C) \\
\text { seeds and hot red } \\
\text { pepper }(R P)\end{array}$ & $\begin{array}{c}\text { F: trans-anethole } \\
\text { BC: thymoquinone, } \\
\text { anethole, carvacrol and } \\
\text { 4-terpinol } \\
\text { RP: Capsaicin }\end{array}$ & 5000 for each & $\begin{array}{l}\text { Corn-soybean } \\
\text { meal-based diet }\end{array}$ & $\begin{array}{l}\text { Lohmann Brown } \\
\text { Lite laying hens } \\
\text { (32-40 weeks) }\end{array}$ & $\begin{array}{c}\uparrow \text { Egg weight, egg } \\
\text { production, egg } \\
\text { mass and } \downarrow \text { FCR } \\
\text { by F and RP }\end{array}$ & [53] \\
\hline Green tea & Polyphenols & 200 & $\begin{array}{l}\text { Corn-soybean } \\
\text { meal-based diet }\end{array}$ & $\begin{array}{l}\text { Hy-line Brown } \\
\text { (65-74 weeks) }\end{array}$ & $\begin{array}{l}\uparrow \text { Egg production } \\
\text { and } \downarrow \text { FCR }\end{array}$ & [95] \\
\hline EOs & $\begin{array}{l}\text { Thymol }(13.5 \%) \text { and } \\
\text { cinnamaldehyde }(4.5 \%)\end{array}$ & $\begin{array}{l}50,100 \text { and } \\
150\end{array}$ & $\begin{array}{l}\text { Corn-wheat- } \\
\text { soybean meal } \\
\text { based diet }\end{array}$ & $\begin{array}{l}\text { Lohmann White } \\
\text { (54-65 weeks) }\end{array}$ & $\begin{array}{c}\text { =Egg production, } \\
\text { egg weight, egg } \\
\text { quality, FI and } \\
\text { FCR }\end{array}$ & [65] \\
\hline
\end{tabular}


Table 2. Cont.

\begin{tabular}{|c|c|c|c|c|c|c|}
\hline Feed Additive & Major Components & $\begin{array}{l}\text { Dose }(\mathrm{mg} / \mathrm{kg} \\
\text { Diet) }\end{array}$ & Diet & Line and Age & Main Findings & References \\
\hline $\begin{array}{c}\text { Echinacea purpurea } \\
\text { powder }\end{array}$ & $\begin{array}{l}\text { Caffeic acid and alkamids, } \\
\text { phenolic acids, } \\
\text { polyacetylenes }\end{array}$ & $\begin{array}{c}2500,5000 \\
7500 \text { and } \\
10,000\end{array}$ & $\begin{array}{l}\text { Corn-soybean } \\
\text { meal-based diet }\end{array}$ & $\begin{array}{l}\text { Leghorn laying } \\
\text { hens (43-53 } \\
\text { weeks) }\end{array}$ & $\begin{array}{l}\uparrow \text { Egg production } \\
\text { and egg mass }\end{array}$ & [96] \\
\hline $\begin{array}{l}\text { Peppermint EO } \\
\text { Thyme EO }\end{array}$ & $\begin{array}{l}\text {-Menthol and menthone } \\
\text {-Thymol, } \gamma \text {-Terpinen and } \\
\rho \text {-Cymene }\end{array}$ & 1000 & $\begin{array}{l}\text { Corn-soybean } \\
\text { meal-based diet }\end{array}$ & $\begin{array}{l}\text { Lohmann LSL-lite } \\
\text { (40-48 weeks) }\end{array}$ & $\begin{array}{c}\uparrow \text { Egg production } \\
\text { and egg mass } \\
\downarrow \text { FCR }\end{array}$ & [97] \\
\hline $\begin{array}{l}\text { Dried grape } \\
\text { pomace }\end{array}$ & Polyphenols & $\begin{array}{c}40,000 \text { and } \\
60,000\end{array}$ & $\begin{array}{l}\text { Corn-soybean } \\
\text { meal-based diet }\end{array}$ & $\begin{array}{l}\text { Bovans laying } \\
\text { hens (80-92 } \\
\text { weeks) }\end{array}$ & $\begin{array}{l}\text { =Live weight, } \\
\text { feed intake, egg } \\
\text { production and } \\
\text { feed efficiency }\end{array}$ & [98] \\
\hline $\begin{array}{l}\text { Fennel }(\mathrm{F}) \text { and } \\
\text { thyme }(\mathrm{T}) \text { extracts }\end{array}$ & $\begin{array}{l}\text { F: anethole, limonene } \\
\text { T:-Thymol, } \gamma \text {-Terpinen } \\
\text { and } \rho \text {-Cymene }\end{array}$ & 40 & $\begin{array}{l}\text { Corn-soybean } \\
\text { meal-based diet }\end{array}$ & $\begin{array}{l}\text { Hy-Line White } \\
\text { (26-38 weeks) }\end{array}$ & $\begin{array}{l}\uparrow \text { Egg weight and } \\
\text { egg mass }\end{array}$ & [99] \\
\hline \multicolumn{7}{|c|}{ Cold stress + Escherichia coli } \\
\hline Curcuma longa & Curcumin & 200 & $\begin{array}{l}\text { Corn-soybean } \\
\text { meal-based diet }\end{array}$ & $\begin{array}{l}\text { Hy-Line Brown } \\
\text { laying hens } \\
(84-90)\end{array}$ & $\begin{array}{c}\text { =Egg production, } \\
\text { egg mass, feed } \\
\text { intake and FCR }\end{array}$ & [100] \\
\hline \multicolumn{7}{|c|}{ Cold stress } \\
\hline Oregano EO & Carvacrol and thymol & $\begin{array}{l}50,100,150 \\
\text { and } 200\end{array}$ & $\begin{array}{l}\text { Corn-soybean } \\
\text { meal-based diet }\end{array}$ & $\begin{array}{c}\text { Semi-heavy } \\
\text { laying hens } \\
\text { (59-71 weeks) }\end{array}$ & $\begin{array}{l}\text { =FCR, egg } \\
\text { production and } \\
\text { egg mass }\end{array}$ & [73] \\
\hline \multicolumn{7}{|c|}{ Heat stress } \\
\hline Grape pomace flour & Polyphenols & $\begin{array}{c}10,000,20,000 \\
\text { and } 30,000\end{array}$ & $\begin{array}{l}\text { Corn-soybean } \\
\text { meal-based diet }\end{array}$ & $\begin{array}{l}\text { Hy-Line lineage } \\
\text { (74-79 weeks) }\end{array}$ & $\uparrow \mathrm{FI}$ & [101] \\
\hline
\end{tabular}

ADG: Average Daily Gain; ADFI: Average Daily Feed Intake; FI: Feed Intake; FCR: Feed Conversion Ratio; $\uparrow:$ increased; $\downarrow$ : decreased; $=$ : equal.

Table 3. Effects of dietary supplementation of PFAs on growth performance of other birds under non-challenging conditions.

\begin{tabular}{|c|c|c|c|c|c|c|}
\hline Feed Additive & Major Components & $\begin{array}{l}\text { Dose (mg/kg } \\
\text { Diet) }\end{array}$ & Diet & Line and Age & Main Findings & References \\
\hline $\begin{array}{l}\text { Grape seed } \\
\text { extract }\end{array}$ & Polyphenols & 100 and 200 & $\begin{array}{l}\text { Corn-soybean } \\
\text { meal-based diet }\end{array}$ & $\begin{array}{c}\text { Duckling (Pekin- } \\
\text { female; } \\
\text { 0-6 weeks) }\end{array}$ & $\begin{array}{c}\uparrow \text { ADG, and final } \\
\text { body weight with } \downarrow \\
\text { FCR }\end{array}$ & [102] \\
\hline Oregano EO & $\begin{array}{c}\text { Carvacrol and thymol } \\
\qquad(85 \%)\end{array}$ & 100 & $\begin{array}{l}\text { Corn-soybean } \\
\text { meal-based diet }\end{array}$ & $\begin{array}{l}\text { Duckling (Cherry } \\
\text { valley; } 0-5 \text { weeks) }\end{array}$ & $=\mathrm{ADG}, \mathrm{FCR}$ & [103] \\
\hline $\begin{array}{l}\text { Eucalyptus } \\
\text { (Eucalyptus } \\
\text { camaldulensis) }\end{array}$ & $\begin{array}{c}\text { p-cymene, 1, } \\
\text { 8-cineole, } \\
\text { b-phellandrene, } \\
\text { spathulenol, cryptone } \\
\text { aldehydes, cuminal, } \\
\text { phellandral, and } \\
\text { a-phellandrene }\end{array}$ & 100 and 200 & NM & $\begin{array}{l}\text { Laying Japanese } \\
\text { quails }\end{array}$ & $=$ Productive traits & [104] \\
\hline Oregano EO & $\begin{array}{l}\text { Thymol (5\%) and } \\
\text { carvacrol (65\%) }\end{array}$ & 150 and 300 & $\begin{array}{l}\text { Corn-soybean } \\
\text { meal-based diet }\end{array}$ & $\begin{array}{c}\text { Duckling (Cherry } \\
\text { valley; } \\
\text { 11-42 days) }\end{array}$ & $\begin{array}{l}=\text { Final body weight, } \\
\text { ADG, FI, and FCR }\end{array}$ & [105] \\
\hline NM & Thymol & $\begin{array}{c}2000,4000 \text { and } \\
6250\end{array}$ & NM & $\begin{array}{l}\text { Quail (Coturnix } \\
\text { japonica; } \\
\text { 85-128 days) }\end{array}$ & $\begin{array}{c}=\mathrm{BWG}, \mathrm{FI}, \text { egg } \\
\text { production, and egg } \\
\text { weight }\end{array}$ & [106] \\
\hline $\begin{array}{c}\text { Leaves of } \\
\text { Astragalus } \\
\text { membranaceus }\end{array}$ & $\begin{array}{c}\text { Polyphenols } \\
\text { (saponins, flavonoids) }\end{array}$ & $\begin{array}{l}10,000,30,000 \\
\text { and } 50,000\end{array}$ & $\begin{array}{l}\text { Corn-soybean } \\
\text { meal-based diet }\end{array}$ & $\begin{array}{l}\text { Japanese quail } \\
\text { (0-35 days) }\end{array}$ & $\uparrow \mathrm{FI}$, and weight gain & [107] \\
\hline $\begin{array}{l}\text { Mentha piperita } \\
\text { (peppermint) }\end{array}$ & Phenolic compounds & $\begin{array}{c}10,000,20,000 \\
30,000 \text { and } \\
40,000\end{array}$ & $\begin{array}{l}\text { Corn-soybean } \\
\text { meal-based diet }\end{array}$ & Quail & $=\mathrm{FI}$ and $\mathrm{ADG}$ & [108] \\
\hline
\end{tabular}

BWG: body weight gain; ADG: Average Daily Gain; ADFI: Average Daily Feed Intake; FI: Feed Intake; FCR: Feed Conversion Ratio; $\uparrow:$ increased; $\downarrow$ : decreased; =: equal. 
Table 4. Effects of dietary supplementation of PFAs on growth performance of broilers under challenging conditions.

\begin{tabular}{|c|c|c|c|c|c|c|c|c|}
\hline \multirow[t]{2}{*}{ Feed Additive } & \multirow[t]{2}{*}{ Major Components } & \multirow{2}{*}{$\begin{array}{c}\text { Dose, } \\
\text { (mg/kg } \\
\text { Diet) }\end{array}$} & \multirow[t]{2}{*}{ Diet } & \multirow[t]{2}{*}{ Age } & \multicolumn{3}{|c|}{$\begin{array}{l}\text { Treatment Effects (\%, } \\
\text { Compared to Control) }\end{array}$} & \multirow[t]{2}{*}{ References } \\
\hline & & & & & BW & ADFI/FI & FCR & \\
\hline \multicolumn{9}{|c|}{ Clostridium perfringens } \\
\hline $\begin{array}{l}\text { Herb: Macleaya } \\
\text { cordata plant }\end{array}$ & $\begin{array}{l}\text { Four specific alkaloids mainly } \\
\text { sanguinarine and protopine }\end{array}$ & 120 & $\begin{array}{l}\text { Corn-soybean } \\
\text { meal-based } \\
\text { diet }\end{array}$ & $15-35 \mathrm{~d}$ & 12.7 & NS & -14.8 & [109] \\
\hline $\begin{array}{c}\text { Plant: Macleaya } \\
\text { cordata } \\
\text { Plant extracts } \\
\text { EOs }\end{array}$ & $\begin{array}{l}\text { Benzo [c]phenanthridine alkaloids } \\
\text { Carvacrol }(4.95 \mathrm{~g} / 100 \mathrm{~g}), \\
\text { cinnamaldehyde }(2.97 \mathrm{~g} / 100 \mathrm{~g}) \text {, and } \\
\text { capsaicin }(1.98 \mathrm{~g} / 100 \mathrm{~g}) \\
\text { Thyme and anise, oregano, carvacol, } \\
\text { yucca extract and cinnamaldehyde }\end{array}$ & $\begin{array}{l}\text { NM } \\
\text { NM } \\
\text { NM }\end{array}$ & $\begin{array}{l}\text { Corn-soybean } \\
\text { meal-based } \\
\text { diet }\end{array}$ & $15-21 \mathrm{~d}$ & $\begin{array}{l}\text { NS } \\
\text { NS } \\
\text { NS }\end{array}$ & $\begin{array}{l}\text { NS } \\
\text { NS } \\
\text { NS }\end{array}$ & $\begin{array}{l}-8.9 \\
-10.0 \\
-11.6\end{array}$ & [74] \\
\hline $\mathrm{EO}$ & $\begin{array}{l}\text { Thymol (25\%) and carvacrol }(25 \%) \text { as } \\
\text { active components }\end{array}$ & $\begin{array}{l}60,120 \text { and } \\
240\end{array}$ & $\begin{array}{l}\text { Wheat- } \\
\text { soybean } \\
\text { meal-based } \\
\text { diet }\end{array}$ & $14-28 \mathrm{~d}$ & NS & NS & NS & [76] \\
\hline \multicolumn{9}{|c|}{ Eimeria } \\
\hline $\begin{array}{l}\text { Herb: Curcuma } \\
\text { longa }\end{array}$ & Curcumin & $\begin{array}{l}100 \text { and } \\
200\end{array}$ & $\begin{array}{c}\text { Corn-soybean } \\
\text { meal-based } \\
\text { diet }\end{array}$ & $12-20 \mathrm{~d}$ & NS & NS & NS & [81] \\
\hline $\begin{array}{l}\text { EOs: cashew nut } \\
\text { shell liquid and } \\
\text { castor oil }\end{array}$ & $\begin{array}{l}\text { Cardanol, cardol, and anacardic acid } \\
\text { Ricinoleic acid }\end{array}$ & 1500 & $\begin{array}{l}\text { Corn-soybean } \\
\text { meal-based } \\
\text { diet }\end{array}$ & $0-42 \mathrm{~d}$ & 2.3 & NS & NS & [77] \\
\hline \multicolumn{9}{|c|}{ Escherichia coli } \\
\hline Resveratrol & Polyphenols & 600 & $\begin{array}{l}\text { Corn-soybean } \\
\text { meal-based } \\
\text { diet }\end{array}$ & $0-42 \mathrm{~d}$ & 6.1 & 2.2 & -3.9 & [84] \\
\hline \multicolumn{9}{|c|}{ Salmonella typhimurium } \\
\hline $\begin{array}{l}\text { Plant: Macleaya } \\
\quad \text { cordata }\end{array}$ & Benzo [c]phenanthridine alkaloids & 5000 & $\begin{array}{l}\text { Corn-soybean } \\
\text { meal-based } \\
\text { diet }\end{array}$ & $8-15 d$ & NS & NS & -11.0 & [85] \\
\hline \multicolumn{9}{|c|}{ Heat-Stress } \\
\hline Plant: Turmeric & Curcumin & 100 & $\begin{array}{l}\text { Corn-soybean } \\
\text { meal-based } \\
\text { diet }\end{array}$ & $21-42 \mathrm{~d}$ & NS & NS & -2.8 & [71] \\
\hline $\begin{array}{l}\text { Herb: Zingiber } \\
\text { officinale }\end{array}$ & $\begin{array}{l}\text { Gingerdiol, gingerol, gingerdione, } \\
\text { and shogaols }\end{array}$ & 2000 & $\begin{array}{l}\text { Corn-based } \\
\text { diet }\end{array}$ & $0-42 \mathrm{~d}$ & 3.3 & NS & 3.0 & [70] \\
\hline $\begin{array}{l}\text { Herb: Artemisia } \\
\quad \text { annua }\end{array}$ & $\begin{array}{l}\text { Phenolics (44.24 mg GAE/g) and } \\
\text { flavonoids (27.8 mg RE/g) }\end{array}$ & 1000 & $\mathrm{NM}$ & $21-42 \mathrm{~d}$ & 8.2 & 4.1 & NS & [69] \\
\hline $\begin{array}{l}\text { Turmeric rhizome } \\
\text { powder }\end{array}$ & Phenolic compounds: curcuminoids & 2000 & $\begin{array}{l}\text { Corn-soybean } \\
\text { meal-based } \\
\text { diet }\end{array}$ & $0-42 \mathrm{~d}$ & 10.6 & NS & 6.9 & [68] \\
\hline
\end{tabular}

GAE: Total phenolic contents were expressed as Gallic Acid Equivalents (mg GAE/g); RE: total flavonoid content was expressed as Rutin Equivalents (mg RE/g); NM: Not Mentioned; NS: Not Significant; BW: Body Weight; ADFI: Average Daily Feed Intake; FI: Feed Intake; FCR: Feed Conversion Ratio.

\subsection{Effects of Dietary PFA Supplementation on Digestibility}

Evaluating digestibility is important as it directly contributes to the animal feed efficiency. However, improving the digestibility is crucial not only for better feed efficiency but also to reduce the amount of undigested feed in the gut, which may favour the occurrence of intestinal imbalances. These imbalances may lead to inflammatory processes and accelerated turnover of intestinal tissue, which results in poorer performance.

Numerous studies have been carried out to study the effects of PFAs inclusion on nutrient digestibility in broiler chickens, laying hens as well as ducks and showed inconsistent results.

The use of either extracts from olive leaves rich in polyphenols [110] or a bioactive olive pomace extract from Olea europaea [62] failed to enhance nutrient apparent total tract digestibility (ATTD) coefficients, or apparent ileal digestibility (AID) of dry matter (DM), 
organic matter (OM), ether extract (EE), and gross energy (GE), respectively. However, the use of EOs such as a blend of carvacrol combined with either cinnamaldehyde and capsicum oleoresin [111] or thymol and limonene in encapsulated forms [44] increased fat digestibility (FD), the AID of crude protein (CP), phosphorus and cysteine. Similarly, a combination of over 30 essential oils and phytogenic compounds increased the digestibility of DM, CP and EE [112].

In laying hens, dietary supplementation of peppermint oil at $0,74,148,222$, and $296 \mathrm{mg} / \mathrm{kg}$ linearly increased digestibility of CP, EE, and phosphorus from 32 to 44 weeks of age [48], while $100 \mathrm{mg} / \mathrm{kg}$ of EOs including thymol $13.5 \%$ and cinnamaldehyde $4.5 \%$ as major active components significantly increased protein and fat digestibility from 54 to 65 weeks of age [65].

An increased nutrient digestibility was also obtained by supplementing meat-type ducks fed high nutrient density diets by a phytogenic blend containing quillaja, anise, and thyme [113]. Similar results were obtained in broiler chickens supplemented with an EO blend, a quillaja saponin blend, or a combination of both phytogenic preparations [114] or a PFA of Aerva lanata, Piper betle, Cynodon dactylon, and Piper nigrum [58]. These authors attributed the digestion-stimulating properties of the PFA basically to piperine, which has been previously reported to stimulate digestion and increase absorption of selenium, vitamin $B$ complex, $\beta$ carotene, and other nutrients.

Possible mechanisms behind improved nutrient digestibility by PFAs supplementation could be attributed to the ability of these feed additives to stimulate appetite, saliva secretion, intestinal mucus production, bile acid secretion, and activity of digestive enzymes such as trypsin and amylase as well as to positively affect the intestinal morphology [58] or to possess an overlapping mode of action including local effects at the intestinal border and systemic alterations of macronutrient metabolism by these feed additives [114].

\subsection{Effects of Dietary PFA Supplementation on Intestinal Microbiota}

It is well-known that farm animal performance is directly linked with gut function and health, which is determined by the continuous interaction among diet, intestinal integrity, gut microbiome and the immune system of chickens. Keeping in view the importance of intestinal microbiome [115], several authors studied the effects of PFAs on the microbiota composition (Table 5). The results showed that these effects are both phytogenic composition and inclusion level dependent [116,117], and can range from neutral with no effect [117-119] up to beneficial [120-125].

The supplementation of a PFA containing the carvacrol as main active compound [124] was shown to modulate the intestinal microbiota more at cecal rather than ileal level by increasing cecal mucosa-associated levels of Bacteroides spp., Clostridium cluster IV, and Clostridium cluster XIVa. This increase could be considered beneficial as Clostridia are not only dominant in ceca [126], but they also contribute to the maintenance of overall gut function, especially through butyrate production [127]. Deinococcus, Bacillaceae and Caulobacteriales were increased in ileal digesta of laying hens fed on a dietary supplementation of EOs, promoting an increase of digestive enzyme activity leading to improved feed utilization efficiency [128]. Moreover, although some authors reported a decrease of the relative abundance of beneficial commensal bacteria such as Lactobacillus by plant extract supplementation [43,129], several others found Clostridiales and/or Lactobacillales to be higher in broilers supplemented with EOs of carvacrol and thymol [130,131] or thymol, eugenol and piperine [121]. More precisely, addition of thymol and carvacrol to the diet changes the host ileum microbial population dynamics by increasing the abundance of L. crispatus and L. agilis, and decreasing L. salivarius and L. johnsonii [131]. L. crispatus is known to be a rod-shaped species of the genus Lactobacillus and is a hydrogen peroxideproducing beneficial microbial species that plays a key role in the protection of the host from infection [132]. A study conducted by Gudiña et al. [133] revealed that a biosurfactant produced by a L. agilis strain showed considerable anti-adhesive activity against $S$. aureus, as well as antimicrobial activity against $P$. aeruginosa, S. aureus and S. agalactiae. Green tea 
and pomegranate have also been proven to modulate the intestinal microbiota [122,134], especially by promoting beneficial bacteria in the intestinal tract $[125,135]$. In a recent study conducted by Perricone et al. [123], broilers receiving a plant extract composed of green tea leaves (Camellia sinensis) and pomegranate rinds (Punica granatum) promoted greater relative abundance of lactic acid bacteria compared to the control group. These results are of particular interest because lactic acid bacteria are recognized to positively affect the intestine by regulating the composition of intestinal microflora, developing intestinal immunity and promoting gut health [136].

Table 5. Effects of dietary PFA supplementation on microbiota of broilers.

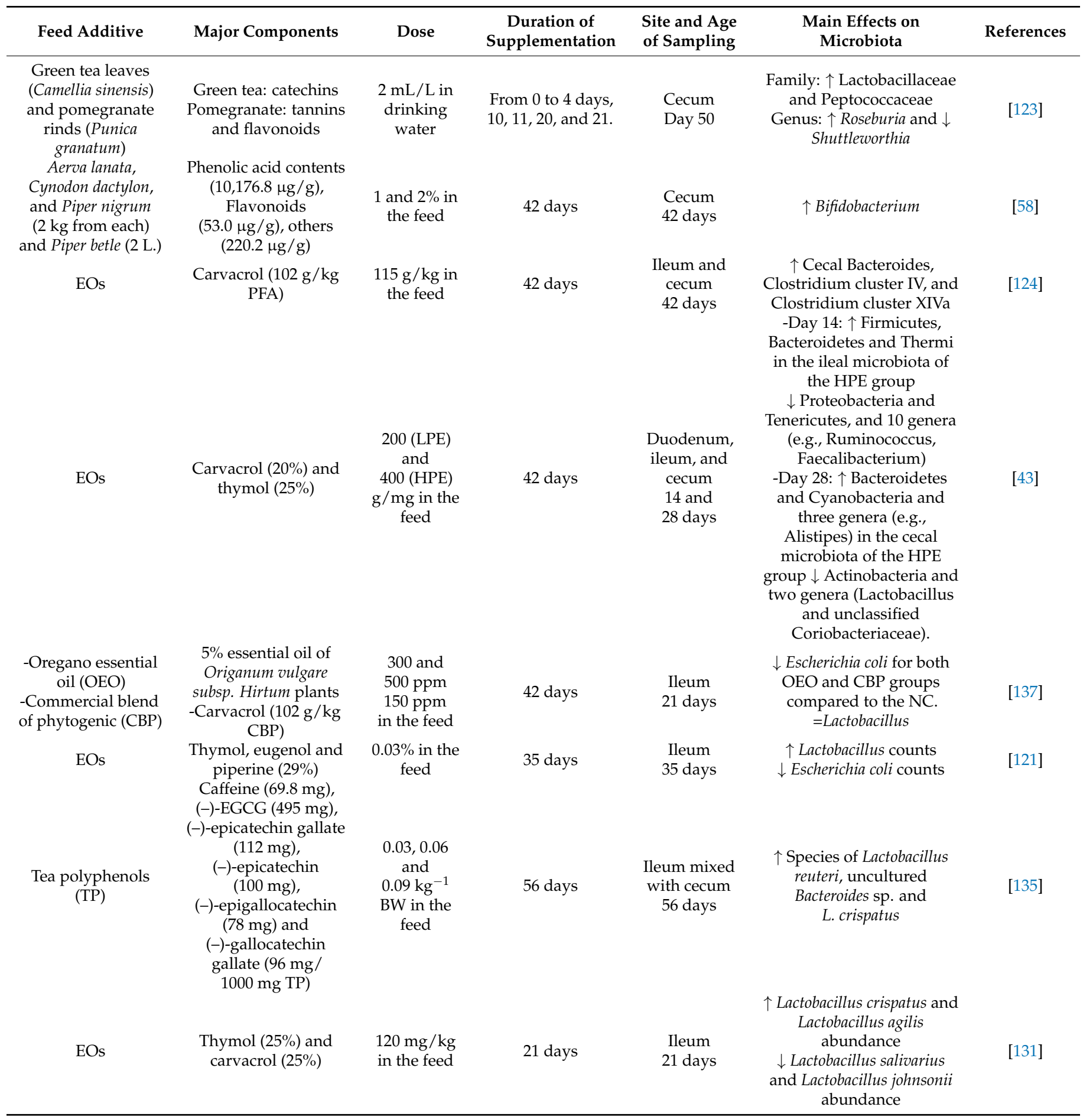


Table 5. Cont.

\begin{tabular}{|c|c|c|c|c|c|c|}
\hline Feed Additive & Major Components & Dose & $\begin{array}{c}\text { Duration of } \\
\text { Supplementation }\end{array}$ & $\begin{array}{l}\text { Site and Age } \\
\text { of Sampling }\end{array}$ & $\begin{array}{l}\text { Main Effects on } \\
\text { Microbiota }\end{array}$ & References \\
\hline EOs & $\begin{array}{l}\text { Equal mixture of } \\
\text { thymol plus carvacrol }\end{array}$ & $\begin{array}{l}100 \mathrm{and} \\
200 \mathrm{mg} / \mathrm{kg} \\
\text { in the feed }\end{array}$ & 42 days & $\begin{array}{l}\text { Duodenum, } \\
\text { jejunum, and } \\
\text { ileum; } \\
24 \text { days }\end{array}$ & $\begin{array}{c}\uparrow \text { Lactobacilli counts } \\
\downarrow \text { Escherichia coli and } \\
\text { Clostridium perfringens } \\
\text { counts with } 200 \mathrm{mg} / \mathrm{kg} \\
\text { Ileum: } \downarrow \text { Escherichia }\end{array}$ & [120] \\
\hline EOs & $\begin{array}{l}\text { Thymol }(25 \%) \text { and } \\
\text { carvacrol }(25 \%)\end{array}$ & $\begin{array}{l}60,120, \text { and } \\
240 \mathrm{mg} / \mathrm{kg} \\
\text { in the feed }\end{array}$ & 28 days & $\begin{array}{l}\text { Ileum and } \\
\text { cecum; } 21 \\
\text { and } 28 \text { days }\end{array}$ & $\begin{array}{c}\text { populations } \\
\text { Cecum: } \downarrow \text { numbers of total } \\
\text { bacteria and Escherichia on } \\
\text { day } 28\end{array}$ & [130] \\
\hline
\end{tabular}

$\uparrow:$ increased; $\downarrow$ : decreased; =: equal.

\subsection{Effects of Dietary PFA Supplementation on Immunity}

An increasing number of studies have shown that health-promoting activities of phytochemicals are attributed to their ability to improve host defence against microbial infection [138]. The immune activating properties of several phytochemicals such as dandelion (Taraxacum officinale), mustard (Brassica juncea) and safflower (Carthamus tinctorius) [139] as well as thistle (Silybum marianum), turmeric (Curcuma longa), reishi mushroom (Ganoderma lucidum), and shiitake mushroom (Lentinus edodes) [140] have been evaluated in vitro using avian lymphocytes and macrophages. In both studies, all extracts inhibited tumour cell growth and stimulated the innate immunity in poultry. These results were further confirmed by several in vivo trials, which are giving increasing evidence that through interactions with immune system, PFAs can modulate immune responses through various mechanisms. Some of the most recent studies are synthesized in Table 6. Dietary immunomodulation is a key to enhance the productivity and immune system integrity of farm animals raised under the absence of antibiotics [141]. One of those mechanisms is the modulation of the expression of the cytokines playing a key role in both the adaptive and the innate immune system [142,143].

Table 6. Effects of dietary PFA supplementation on immunity of broilers.

\begin{tabular}{|c|c|c|c|c|c|}
\hline Feed Additive & Major Components & $\begin{array}{l}\text { Dose, }(\mathrm{mg} / \mathrm{kg} \\
\text { Diet) }\end{array}$ & $\begin{array}{l}\text { Experimental } \\
\text { Conditions }\end{array}$ & Immune Response & References \\
\hline $\begin{array}{l}\text { Curcumin } \\
\text { EOs (PHY) }\end{array}$ & $\begin{array}{c}\text { Curcumin }(72 \% ; \mathrm{CU}) \\
\text { carvacrol }(21.55 \mathrm{mg} / \mathrm{g}), \\
\text { thymol }(18.76 \mathrm{mg} / \mathrm{g}) \text { and } \\
\text { cinnamaldehyde } \\
(27.62 \mathrm{mg} / \mathrm{g}) \text { of PHY }\end{array}$ & $\begin{array}{c}50 \\
100\end{array}$ & $\begin{array}{l}\text { Corn-soybean } \\
\text { meal-based diet }\end{array}$ & $\begin{array}{l}\downarrow \text { Total leukocyte and } \\
\text { heterophils number in the CU } \\
\text { and PHY + CU groups, } \downarrow \\
\text { lymphocytes in the CU group }\end{array}$ & [144] \\
\hline EOs & $\begin{array}{l}\text { Oregano oil (50 g), } \\
\text { carvacrol }(10 \mathrm{~g}) \text {, thyme oil } \\
(33.33 \mathrm{~g}), \text { eucalyptus oil } \\
(50 \mathrm{~g}), \text { thymol }(5 \mathrm{~g}), \\
\text { eucalyptol }(10 \mathrm{~g}), \text { and } \\
\text { acacia (Arabic gum) } \\
\text { surfactant ( } 27 \mathrm{~g}) \text { in water } \\
\text { up to } 1 \mathrm{~L}\end{array}$ & 500 & $\begin{array}{l}\text { Challenge with } \\
\text { virulent Newcastle } \\
\text { disease virus+ vaccin } \\
\text { against Newcastle } \\
\text { disease (ND), the } \\
\text { avian influenza (AI), } \\
\text { infectious bronchitis } \\
\text { (IB), and infectious } \\
\text { bursal disease (IBD) }\end{array}$ & $\begin{array}{c}\downarrow \text { Hemagglutination } \\
\text { inhibition and viral shedding } \\
\text { titres } 1 \mathrm{wk} \text { after challenge } \\
\uparrow \text { ELISA antibody titre for } \\
\text { IBD virus at the } 28 \text { th } d \text { of age }\end{array}$ & [145] \\
\hline Resveratrol & Polyphenols & 300 and 600 & $\begin{array}{l}\text { Corn-soybean } \\
\text { meal-based diet and } \\
\text { chickens challenged } \\
\text { with Escherichia coli }\end{array}$ & $\begin{array}{l}\uparrow \text { Total Ig and IgG at d22 and } \\
\text { total Ig and IgM at d } 35\end{array}$ & [84] \\
\hline $\begin{array}{l}\text { Cashew nut shell } \\
\text { liquid and castor oil }\end{array}$ & $\begin{array}{l}\text { Cardanol, cardol, and } \\
\text { anacardic acid } \\
\text { Ricinoleic acid }\end{array}$ & 1500 & $\begin{array}{l}\text { Broilers challenged } \\
\text { with Eimeria spp. }\end{array}$ & $\begin{array}{c}\uparrow \text { Gene expression of TNF- } \alpha \text {, } \\
\text { IL- } 6 \text { and IFN- } \gamma \text { and } \downarrow \\
\text { expression of IL- } 1 \text { and COX-2 }\end{array}$ & [146] \\
\hline Yucca schidigera & Saponins & 250 & $\begin{array}{l}\text { Corn-soybean } \\
\text { meal-based diet } \\
\text { Mixed Eimeria } \\
\text { challenge }\end{array}$ & $\begin{array}{l}\text { =Lymphocyte percentages to } \\
\text { that of unchallenged birds on } \\
\text { d7 p.i }\end{array}$ & [80] \\
\hline
\end{tabular}


Table 6. Cont.

\begin{tabular}{|c|c|c|c|c|c|}
\hline Feed Additive & Major Components & $\begin{array}{l}\text { Dose, }(\mathrm{mg} / \mathrm{kg} \\
\text { Diet) }\end{array}$ & $\begin{array}{l}\text { Experimental } \\
\text { Conditions }\end{array}$ & Immune Response & References \\
\hline EOs & $\begin{array}{c}\text { Carvacrol }(5 \%), \\
\text { cinnamaldehyde }(3 \%), \\
\text { and capsicum oleoresin } \\
(2 \%)\end{array}$ & 100 & $\begin{array}{l}\text { Two control diets } \\
\text { based on either } \\
\text { wheat or maize }\end{array}$ & $\downarrow$ CD40LG, IFN- $\gamma$ and IL-6. & [86] \\
\hline EOs & $\begin{array}{l}\text { Cinnamon bark oil (CNO) } \\
\text { Clove bud oil (CLO) } \\
\text { Ajwain seed oil (AJO) }\end{array}$ & $\begin{array}{l}300 \\
600 \\
400\end{array}$ & $\begin{array}{c}\text { Corn-soybean } \\
\text { meal-based diet. } \\
\text { Broilers vaccinated } \\
\text { against NDV at } 5 \text { and } \\
18 \mathrm{~d} \text { of age, and IBDV } \\
\text { at } 14 \mathrm{~d} \text { of age. }\end{array}$ & $\begin{array}{c}\uparrow \text { Antibody titres against } \\
\text { NDV vaccine with CNO and } \\
\text { CLO at } 35 \mathrm{~d} \text { of age }\end{array}$ & [147] \\
\hline EOs & $\begin{array}{l}\text { Carvacrol, thymol and } \\
\text { cinnamic aldehyde }\end{array}$ & 5000 and 10,000 & $\begin{array}{l}\text { Corn-soybean } \\
\text { meal-based diet. }\end{array}$ & $\begin{array}{c}\uparrow \text { Total erythrocyte counts, } \\
\text { hemoglobin content and } \downarrow \\
\text { leucocyte count }\end{array}$ & [42] \\
\hline Thyme powder & $\begin{array}{c}\text { Major EOs (thymol } \\
(50.48 \%), \gamma \text {-terpinene } \\
(11.03 \%), \text { P-cymene } \\
(9.77 \%), \text { and carvacrol } \\
(4.30 \%)) \text {, phenolic acids } \\
(\text { salicylic acid } \\
(2450.03 \text { ppm), ellagic } \\
\text { acid (1240.42 ppm)) and } \\
\text { flavonoid compounds }\end{array}$ & $\begin{array}{l}2000,5000 \text { and } \\
8000\end{array}$ & $\begin{array}{l}\text { Corn-soybean } \\
\text { meal-based diet. }\end{array}$ & $\begin{array}{c}\uparrow \text { Lymphocytes, white blood } \\
\text { cells, and IgG. } \\
\downarrow \text { TNF- } \alpha \text {, IFN- } \gamma \text {, NF-kBP50 by } \\
\text { all the doses. } \\
\downarrow \text { IL- } 6 \text { by the dose of } 8000\end{array}$ & [87] \\
\hline $\begin{array}{c}\text { Allium hookeri }(\mathrm{AH}) \\
\text { roots } \\
\text { Fermented root }\end{array}$ & Phenols & $\begin{array}{l}10,000 \text { and } 50,000 \\
\text { for both }\end{array}$ & $\begin{array}{l}\text { Corn-soybean } \\
\text { meal-based diet. } \\
\text { LPS-induced young } \\
\text { broiler chicken }\end{array}$ & $\begin{array}{l}\downarrow \text { IL- } 1 \mathrm{~b} \text { with } 1 \% \text { AH root and } \\
5 \% \text { fermented root, TNFSF15 } \\
\text { expression with fermented } \\
\text { root }(1 \% \text { and } 5 \%) \text {, and IL- } 8 \\
\text { with } 1 \% \text { fermented root } \\
\text { supplementation }\end{array}$ & [148] \\
\hline EOs & Oregano: (5\%) & 300 & $\begin{array}{l}\text { Corn-soybean } \\
\text { meal-based diet. }\end{array}$ & $\begin{array}{l}\uparrow \text { Secondary antibody titer } \\
\text { and IgG titer, } \downarrow \text { H/L ratio }\end{array}$ & [88] \\
\hline Artemisia апnиа & $\begin{array}{c}\text { Phenolics (44.24 mg } \\
\text { GAE/g) and flavonoids } \\
\text { (27.8 mg RE/g) }\end{array}$ & 1000 & Heat-stressed broilers & $\uparrow$ Intestinal SIgA and IgG & [69] \\
\hline Turmeric rhizome & $\begin{array}{l}\text { Carvacrol }(60.2 \%) \text { and } \\
\text { thymol }(4 \%)\end{array}$ & $\begin{array}{l}50 \text { and } 100 \text { in } \\
\text { water }\end{array}$ & $\begin{array}{l}\text { Corn-soybean } \\
\text { meal-based diet and } \\
\text { broilers kept under } \\
\text { chronic heat stress } \\
\text { Corn-soybean } \\
\text { meal-based diet and } \\
\text { broilers vaccinated } \\
\text { with inactivated } \\
\text { avian influenza and } \\
\text { Newcastle disease } \\
\text { (NDV) }\end{array}$ & $\begin{array}{l}\uparrow \text { Antibody titer against NDV } \\
\text { and avian influenza virus }\end{array}$ & [149] \\
\hline
\end{tabular}

SIgA: secretory immunoglobulin A; p.i.: post infection; $\downarrow$ : increased; $\uparrow:$ decreased: =: equal.

A study conducted by Pirgozliev et al. [86] demonstrated that broilers under nonchallenging conditions, supplemented by a commercial blend comprising $5 \%$ carvacrol, $3 \%$ cinnamaldehyde, and $2 \%$ capsicum oleoresin showed downregulated IFN- $\gamma$ and IL-6 cytokines indicating a lower inflammation level than those in the other groups.

In broilers under challenging conditions such as necrotic enteritis, Lee et al. [150] showed that a mixture of Capsicum and turmeric oleoresins reduced intestinal IL-8, lipopolysaccharide-induced TNF- $\alpha$ factor (LITAF), IL-17A and IL-17F mRNA levels. Similarly, the expression of pro-inflammatory cytokines was reduced by the supplementation of Allium hookeri in LPS-induced young broiler chickens [148] and thyme powder in broilers without any challenge [87]. Nonetheless, a blend of cashew nut shell liquid (CNSL) and castor oil modulated the inflammatory response of broiler chickens against Eimeria spp. by increasing gene expression of TNF- $\alpha$, IL- 6 and IFN- $\gamma$ and reducing expression of IL-1 and COX-2, one week post-infection [146] These authors concluded that although inflammation is a highly undesirable phenomenon owing to its costly effects on animal production efficiency, this increased inflammatory response observed in challenged birds 
treated with CNSL-castor oil was necessary to help the immune system to effectively fight against coccidiosis and other pathogenic bacteria to prevent intestinal dysbiosis.

Other authors have rather evaluated the levels of SIgA, IgM, and IgG, three major classes of immunoglobulin in chickens that play key roles in the maintenance of immunity [151]. SIgA is involved in the protection and homeostatic regulation of intestinal mucosal epithelia by limiting the access of numerous microorganisms and mucosal antigens, while IgG directly contributes to an immune response including neutralization of toxins and viruses. A decrease of SIgA and IgG observed in the jejunum mucosa of heat-stressed broilers was counteracted by the supplementation of enzymatically treated Artemisia annua [69]. Similarly, an increase of $\operatorname{IgG}$ was observed by the supplementation of thyme powder [87] and EOs of oregano [88] in broilers raised under normal conditions or by the supplementation of resveratrol in chickens challenged with Escherichia coli [84].

The effect of PFAs, especially EOs, on the immune response to some viral vaccines in broiler chickens was also evaluated in several studies. A mixture containing oregano oil (50 g), carvacrol (10 g), thyme oil (33.33 g), eucalyptus oil (50 g), thymol (5 g), eucalyptol $(10 \mathrm{~g})$, and acacia (Arabic gum) surfactant $(27 \mathrm{~g})$ supplemented through the drinking water at a dose of $0.5 \mathrm{~mL} / \mathrm{L}$ showed an immune-stimulating response to Newcastle disease (ND) and infectious bursal disease (IBD) vaccines, antiviral effect against ND virus, especially if administered before the challenge [145].

The supplementation of cinnamon bark oil (CNO) [147,152], clove bud oil (CLO) and ajwain seed oil (AJO) [147], cinnamaldehyde combined with formic acid [147] and Origanum essential oil containing $60.2 \%$ carvacrol and $4 \%$ thymol [149] enhanced immune response against NDV in broiler chickens. However, antibody titres against avian influenza virus and NDV were unaffected by cinnamon bark powder supplementation ( 2 and $4 \mathrm{~g} / \mathrm{kg}$ diet) [153] or clove EO $(0.15,0.30$ and $0.45 \mathrm{~g} / \mathrm{kg})$ [154], and antibody titres against NDV vaccine and sheep red blood cells were not significantly influenced by the supplementation of EOs mixture obtained from anise, oregano, and citrus peel in broiler chickens [155].

The supplementation of black cumin (Nigella sativa) combined with Echinacea enhanced the immune response after AI-H9N2 vaccination and reduced the pathogenicity of infection in dexamethasone-stressed chickens [156]. The immune-modulating effects of black cumin may be attributed to pharmacologically active constituents, such as thymol, nigellidine, nigellimine, thymoquinone, dithymoquinone and thymohydroquinone [157] which are able to induce pharmacological effects against antigenic challenge [158].

\subsection{Effects of Dietary PFA Supplementation on Blood Biochemical Parameters and Oxidant Status}

Several authors studied the effect of PFA supplementation on the serum biochemical indicators that help to display the nutrient's metabolism and body physiological state [159]. Serum lipid parameters have been shown to be reduced in broiler chickens by the supplementation of lavender EO (cholesterol and LDL-C; [160]), Pulicaria gnaphalodes powder (cholesterol and triglycerides; [59]) and a mixture of oregano, anise and citrus EOs (cholesterol; [161]). Serum cholesterol has been also reduced in laying hens supplemented by EOs [162], either black cumin or hot red pepper [53], peppermint oil [48] or nettle Urtica cannabina [64].

The mechanisms explaining the hypocholesterolemic effect of PFAs may be associated to the reduced activity of enzymes involved in lipid metabolism including 3-hydroxy-3methylglutaryl-CoA (HMG-CoA) reductase (enzyme associated with cholesterol synthesis), cholesterol-7 hydroxylase fatty acid synthase and pentose phosphate pathway [147,163].

The supplementation of PFAs has been also shown to improve the antioxidant status of broilers [164-166]. A recent study performed by Paraskeuas et al. [39] revealed that a mixture of menthol, anethol and eugenol increased blood plasma total antioxidant capacity (TAC) in a linear pattern, corroborating the results previously obtained by other authors $[167,168]$. The activity of superoxide dismutase (SOD) and glutathione peroxidase (GSH-Px), enzymes considered as one of the defensive mechanism of the body against the oxidative stress [169], was increased by supplementing broilers by lavender EO [160] and 
laying hens by star anise oil [45] and grape pomace [101]. Malondialdehyde (MDA) levels were also decreased in laying hens supplemented by grape pomace [98] and star anise oil [45].

\subsection{Effects of Dietary PFA Supplementation on Meat, Internal and External Egg Quality}

Benefits of dietary PFA supplementation on the quality and shelf-life of meat products are still ambiguous. Although the supplementation of a PFA based on EOs of carvacrol, thymol and cinnamic aldehyde was unable to prevent broiler meat lipid peroxidation caused by freezing temperatures [42], other authors reported increased antioxidant levels and reduction of lipid peroxidation by Nigella sativa seeds [52], turmeric [57], curcuminoids [56] as well as herbal components containing curcumin, carvacrol, thymol and cinnamaldehyde [144]. Manipulating lipid peroxidation has been shown to be, in part, achieved through modulating the profile of meat fatty acids by PFAs supplementation. Total saturated fatty acid (SFA) levels were reduced and monounsaturated/polyunsaturated fatty acid (MUFA/PUFA) levels were increased by PFAs supplementation [56,144,170,171]. Particularly, SFA such as lauric, stearic, myristic and palmitic acid are undesirable due to their hypercholesterolemic properties in the form of LDL $[144,170]$. On the contrary, omega 3 and 6 fatty acids play key roles in human nutrition, being precursors of principal molecules involved in the regulation of the cardiovascular and immune system including eicosanoids, prostaglandins, leukotrienes and thromboxanes [144].

External and internal egg quality has been also shown to be influenced by PFAs supplementation. Eggshell thickness was increased by supplementation of herbal EOs mixture [162], peppermint oil [48] and nettle Urtica cannabina [64]. A possible mechanism behind these positive effects is the ability of PFAs such as EOs to improve uterine health and increase calcium storage as well as pancreatic secretions, resulting in the enhancement of nutrient digestion and consequently the improvement in eggshell and egg quality [48].

Regarding internal quality, the supplementation of PFAs such as black cumin or red pepper [53], star anise (Illicium verum Hook.f.) EO [45] grape pomace flour [101] and curcumin [100] has been shown to reduce egg lipid peroxidation and increase its antioxidant levels, thereby, generating internal stability of the stored eggs and contributing to extending egg shelf life. The Haugh unit score, known as an indicator of egg freshness and is related to shelf life, has been also shown to be increased by PFAs supplementation $[46,48,63]$. Similar to meat, egg yolk cholesterol content has been shown to be reduced by PFAs supplementation $[48,64]$. Although the egg yolks of laying hens supplemented with Citrullus lanatus EOs showed increased total PUFA and n- 6 fatty acids and reduced n-3 fatty acids [46], nettle Urtica cannabina supplementation increased total n-3 PUFA concentration while reducing the ratio of $n-6 / n-3$ [64].

\section{Challenges and Prospective of Using PFAs in Animal Nutrition \\ 5.1. Challenges of Using PFAs in Animal Nutrition}

Performing systematic and comprehensive studies evaluating the efficacy and safety of PFAs is still difficult due to their complex composition [172]. In addition, inconsistency in the obtained results may be attributed to several factors related either to the enormous variability per se of PFA, including source and bioactive compounds of the PFAs which may depend on the plant, botanical origin growing locations, manufacturing methods, the storage conditions, and the effective dose; or to the environmental conditions, management and rearing conditions (challenge vs. no challenge and differences in the underlying microbial challenge if applied, age, genetics... [22,172]. Some authors also reported that the appropriate minimum inhibitory concentration (MIC) for most phytogenic compounds is higher than the level considered as cost-effective [172]. On the other hand, various phytogenic compounds such as EOs can evaporate rapidly due to their volatile and reactive nature, resulting in largely varied concentrations in the final feed additive. Their effectiveness in animals may also be affected by several conditions during production processes and storage. Thus, ensuring their stability presents a difficulty, as do maintaining their 
biological activity and masking their strong odour [173]. Moreover, mutual interactions with other substance from feed matrix have been reported such as lower biological effects of PFA present in fibrous diets or high protein diets [174]. Several phytogenic compounds have been also shown to be largely absorbed in the upper GIT, meaning that without proper protection, the majority would not reach the lower gut where they would exert their major functions. In this context, a study conducted by Hafeez et al. [44] showed that the benefits of supplementing the broiler diet with a mixture of encapsulated EOs were higher than the tested PFA in powdered, non-protected form.

Therefore, novel delivery technologies have been developed to protect PFAs from the degradation and oxidation process during feed processing and storage, ease the handling, allow a slower release and target the lower GIT [173]. Among these techniques, microencapsulation is gaining an increasing interest where various carrier types including polymer-based particles such as polysaccharide-protein carriers and lipid-based particles such as vegetable oils and liposomes were tested. The advantages and disadvantages of both carrier materials from the encapsulation efficiency, loading capacity, and release kinetics viewpoints were previously discussed in the literature. Although advantages of polysaccharide-protein carriers include their mechanical and thermal stabilities, nutritional quality, low cost, and easy preparation procedure, they present low encapsulation efficiency, loading capacity, and release efficiency in small intestine [175]. As for lipid-based particles, they are characterized by high encapsulation efficiency, loading capacity, and release efficiency in the small intestine. However, their disadvantages include low mechanical and thermal stabilities [173]. Moreover, liposomes cannot be used for large scale production owing to complex preparation procedures, reduced production capacity, and higher cost [176].

Despite some studies showed some positive effects on chickens by using single EO, several others have rather chosen the combination of various EOs and their isolated components to take advantages from their synergistic effects [2,177]. Indeed, synergistic interactions are of great importance because they enhance the antimicrobial and antioxidant activity by maximizing the efficiencies of the combined agents in the best possible manner which results in several fold reduction in the required doses of EOs applied in situ and thereby lowering their organoleptic impacts. Interestingly, blends containing hydrocarbons and phenylpropanoids (e.g., cinnamaldehyde, eugenol, carvacrol, and capsicum oleoresin) in combination with other components were reported to enhance the bioactivities of these mixtures [178]. A special attention was placed on the interaction of phenolic monoterpenes (thymol, carvacrol) and phenylpropanoids (eugenol) with other groups of components, particularly with other phenols, phenylpropanoids and monoterpenes alcohols, whereas monoterpenes and sesquiterpenes hydrocarbons were used to a lesser extent [178]. Moreover, combining phenolics with monoterpenes alcohols has been reported to produce synergistic effects on several microorganisms, in particular, the combination of phenolics (thymol with carvacrol, and both components with eugenol) were synergistically active against Escherichia coli strains [178]. In ruminants, these combinations were the most effective dietary supplementation options that showed ruminal antimicrobial advantages to modulate the ruminal fermentation pathways $[179,180]$.

\subsection{Prospective of PFAs in Animal Nutrition: Combination of EOs with OAs}

It has been reported that feed additives with different functions and complementary mode of actions hold the most promising solution to replace antibiotics in animal feed mainly for three reasons: (i) all the beneficial effects of antibiotics are unlikely to be covered by an individual alternative; (ii) some alternatives possess a synergetic effect that may decrease the required dose considered as cost-effective; (iii) substituting the antibiotics must be an integrated approach that includes feeding, management and biosecurity rather than a supplementation of feed additives alone [172].

As for the synergy between feed additives, the combined use of hydrophobic EOs with lipophilic OAs has been considered the most promising method to substitute antibiotics 
for the potential synergistic and additive beneficial effects on the intestinal health and growth performance compared with individual EOs or OAs [181,182]. Some results of recent studies are illustrated in Table 7 . The main mode of action linked to the synergic effects of OAs and EOs may be the modulation of the intestinal microbiota. However, the antimicrobial activities depend on the gram staining of bacteria as Gram-negative (G-) bacteria differ from Gram-positive $(\mathrm{G}+)$ bacteria with the respect to the structure of the cell wall. The cell walls of G+ bacteria are $90-95 \%$ composed of peptidoglycan allowing hydrophobic molecules (EOs) to easily penetrate the cells, acting on both the cell walls and the cytoplasm, causing a disruption of the structure and function of bacteria cell membranes [183]. This increase of the bacterial membrane permeability could facilitate the influx of OAs into the cytoplasm due the lipophilic nature of their undissociated form, disturbing the proton and associated anion concentrations in the cytoplasm [182]. As for phenolic compounds, once inside the cell, they can interfere with enzymes involved in the production of energy at lower concentrations and denature proteins at higher concentration. However, G- bacteria possess a different composition as their peptidoglycan layer is only 2-3 mm thick and composes only $20 \%$ of the dry weight of the cell. An outer membrane comprised of a double layer of phospholipids firmly linked by Braun's lipoprotein to the inner membrane, lies outside of the peptidoglycan layer, making G- bacteria less permeable by providing an extra layer of protection and thus more resistant to EOs than the G+ bacteria [183]. Moreover, the "quid" provided by the core polysaccharides and the O-side chain allows these bacteria to be more resistant to EOs and other natural extracts possessing anti-microbial activities. Although the antimicrobial properties of long chain fatty acids (LCFA) are related to their potential to incorporate themselves into target membranes of $\mathrm{G}+$ bacteria, promoting leakage of cellular protons and ions due to their lipophilic nature, the lipopolysaccharide (LPS) layer in the cell wall of G- bacteria prevents medium chain fatty acids (MCFA) and LCFA from crossing the cell membrane. Moreover, G- bacteria are also able to assimilate MCFA and LCFA into the cell and subsequently metabolize them per the $\beta$-oxidation cycle (i.e., Escherichia coli) and utilize short chain fatty acids (SCFA) as energy sources (i.e., Salmonella, Escherichia coli). These differences in the cell membrane compositions make EOs more powerful in the control of $\mathrm{G}+$ bacteria compared to $\mathrm{G}-$ ones. However, OAs are reported to be more effective against $\mathrm{G}-$ bacteria than EOs [184] as small hydrophilic solutes of OAs are able to pass through the membrane via porin proteins but not the hydrophobic polyphenol molecules [172].

Table 7. Application of mixtures of phytogenics and organic acids with major physiological responses in poultry.

\begin{tabular}{|c|c|c|c|}
\hline Mixture of OA+EO & Study Design & Main Findings & Reference \\
\hline $\begin{array}{c}\text { Citric }(25 \%) \text { and sorbic } \\
(16.7 \%) \text { acids, thymol }(1.7 \%), \\
\text { and vanillin }(1.0 \%)\end{array}$ & $\begin{array}{l}\text { Type: male breeder chickens } \\
\text { Dose: } 500 \mathrm{~g} / \text { metric ton diet } \\
\text { Form: microencapsulated } \\
\text { Duration: } 15 \text { days } \\
\text { Conditions: without challenge }\end{array}$ & $\begin{array}{c}\text {-Increased Lactobacilliaceae, Clostridiaceae and } \\
\text { Ruminococcaceae abundance } \\
\text {-Decreased Staphylococcaceae, }\end{array}$ & [185] \\
\hline $\begin{array}{c}\text { Thyme }(4 \%) \text {, carvacrol }(4 \%) \\
\text { hexanoic acid }(0.5 \%), \\
\text { benzoic acid }(3.5 \%) \text { and } \\
\text { butyric acid }(0.5 \%)\end{array}$ & $\begin{array}{c}\text { Type: male Arbor Acres broiler } \\
\text { chickens } \\
\text { Dose: } 500 \mathrm{mg} / \mathrm{kg} \text { diet } \\
\text { Form: Encapsulated } \\
\text { Duration: } 42 \text { days } \\
\text { Conditions: Eimeria spp. and } \\
\text { Clostridium perfringens }\end{array}$ & $\begin{array}{c}\text {-Improved FCR } \\
\text {-Higher villus height and villus height/crypt } \\
\text { depth ratio. } \\
\text {-Reduced intestinal C. perfringens counts, liver } \\
\text { C. perfringens carriage, and gut lesion scores. } \\
\text {-Reduced serum fluorescein isothiocyanate } \\
\text { dextran (FITC-D) concentrations. } \\
\text {-Upregulated claudin-1, IGF-2 and A20 } \\
\text { mRNA expression. } \\
\text {-Downregulated TRAF-6, TNFSF15 and } \\
\text { TOLLIP mRNA levels }\end{array}$ & [181] \\
\hline
\end{tabular}


Table 7. Cont.

\begin{tabular}{|c|c|c|c|}
\hline Mixture of OA+EO & Study Design & Main Findings & Reference \\
\hline $\begin{array}{l}\text { Citric }(25 \%, \text { as fed }) \text { and } \\
\text { sorbic acids }(16.7 \% \text {, as fed }), \\
\text { thymol }(1.7 \% \text {, as fed) and } \\
\text { vanillin }(1 \% \text {, as fed })\end{array}$ & $\begin{array}{c}\text { Type: Male Ross } 308 \text { broiler } \\
\text { chickens } \\
\text { Dose: } 5 \mathrm{~g} / \mathrm{kg} \text { diet } \\
\text { Form: Encapsulated } \\
\text { Duration: } 47 \text { days } \\
\text { Conditions: without challenge }\end{array}$ & $\begin{array}{c}\text {-Improved growth performances } \\
\text {-Improved gut morphology } \\
\text {-Microbial control against Clostridium } \\
\text { perfringens, Enterobacteriaceae, Enterococci and } \\
\text { Mesophilic bacteria }\end{array}$ & [186] \\
\hline $\begin{array}{l}\text { Fumaric, sorbic, malic, and } \\
\text { citric acids, thymol, vanillin, } \\
\text { and eugenol }\end{array}$ & $\begin{array}{l}\text { Type: Cobb } 500 \text { male broilers } \\
\text { Dose: } 300 \mathrm{~g} / \mathrm{t} \text { diet } \\
\text { Form: Protected } \\
\text { Duration: } 42 \text { days } \\
\text { Conditions: Eimeria spp. and } \\
\text { Clostridium perfringens }\end{array}$ & $\begin{array}{c}\text {-Greater body weight gain } \\
\text {-Higher apparent ileal nutrient and energy } \\
\text { digestibility } \\
\text {-Improved intestinal integrity with lower } \\
\text { blood fluorescein isothiocyanate-dextran } \\
\text { concentration } \\
\text {-Improved intestinal macroscopic and } \\
\text { histologic alterations } \\
\text {-Greater expression of MUC2, CLDN1, and } \\
\text { OCLN genes }\end{array}$ & [182] \\
\hline $\begin{array}{l}\text { Citric andsorbic acids, } \\
\text { thymol, and vanillin }\end{array}$ & $\begin{array}{l}\text { Type: By-product breeder chicks } \\
\text { Dose: } 500 \mathrm{~g} / \text { metric ton diet } \\
\text { Form: Microencapsulated } \\
\text { Duration: } 4 \text { days } \\
\text { Conditions: Without challenge }\end{array}$ & $\begin{array}{l}\text {-Enhanced in vitro functional activity of } \\
\text { peripheral blood leukocytes (degranulation, } \\
\text { oxidative burst, and nitric oxide production) }\end{array}$ & [187] \\
\hline $\begin{array}{l}\text { Sorbic acid }(200 \mathrm{~g} / \mathrm{kg}) \text {, } \\
\text { fumaric acid }(200 \mathrm{~g} / \mathrm{kg}) \text {, and } \\
\text { thymol }(100 \mathrm{~g} / \mathrm{kg})\end{array}$ & $\begin{array}{l}\text { Type: Roman laying hens } \\
\text { Dose: } 150 \text { and } 300 \mathrm{mg} / \mathrm{kg} \text { diet } \\
\text { Form: Encapsulated } \\
\text { Duration: } 21-30 \text { weeks } \\
\text { Conditions: Without challenge }\end{array}$ & $\begin{array}{l}\text {-Increased laying rate with } 150 \mathrm{mg} / \mathrm{kg} \text {. } \\
\text {-A linear increase in ileal villus height. } \\
\text {-Increased mRNA relative expression of } \\
\text { aminopeptidase, sodium-glucose } \\
\text { cotransporter } 1 \text {, and Na+-independent } \\
\text { neutral amino acid transporter in duodenum } \\
\text { and glucose transporter } 2 \text { in jejunum with } \\
\quad 300 \mathrm{mg} / \mathrm{kg} \text {. } \\
\text {-Higher mRNA relative expression ofmucin-2 } \\
\text { in ileum with } 300 \mathrm{mg} / \mathrm{kg} \text {. } \\
\text {-Linear decrease of the secretory } \\
\text { immunoglobulin in ileum A. }\end{array}$ & [188] \\
\hline
\end{tabular}

The mechanism of inhibition to microorganisms by OAs may be affected by several factors, including the reduction in $\mathrm{pH}$, the ratio of the un-disassociated form of the acid, chain length, degree of branching and cell physiology/metabolism [189]. Indeed, the lipophilic nature of weak organic acid allows them to easily penetrate the plasma membrane and thus acidify the cell's interior eventually killing the bacterium [172].

\section{Conclusions}

This review tried to gather the most recent available scientific information regarding the use of phytogenics in poultry nutrition along with their beneficial effects on performance, digestibility, microbiota, immune response, oxidant status, as well as egg and meat quality. Several studies have reported the promising effects of these feed additives when combined together or with organic acids; however, extra attention should be focused on the selection of active compounds to form potentially effective blends. Moreover, choosing the appropriate technique of protection as well as types and physiochemical properties of wall materials are the most critical aspects governing efficiency by controlling both the timing and location of the release of active compounds.

Author Contributions: N.A.: Gathering information and writing the manuscript. J.F.P. and D.S.-O.: reviewing and editing the paper. All authors have read and agreed to the published version of the manuscript. 
Funding: N.A. was funded by an FI Ph.D. grant from the Agència de Gestió d'Ajuts Universitaris i de Recerca de la Generalitat de Catalunya (2018FI_B_01070).

Data Availability Statement: Not applicable.

Conflicts of Interest: The authors declare no conflict of interest.

\section{References}

1. Ricke, S.C.; Dittoe, D.K.; Richardson, K.E. Formic acid as an antimicrobial for poultry production: A review. Front. Vet. Sci. 2020, 7,1-13. [CrossRef]

2. Zhai, H.; Liu, H.; Wang, S.; Wu, J.; Kluenter, A.M. Potential of essential oils for poultry and pigs. Anim. Nutr. 2018, 4, 179-186. [CrossRef]

3. Al-Khalaifah, H.S. Benefits of probiotics and/or prebiotics for antibiotic-reduced poultry. Poult. Sci. 2018, 97, 3807-3815. [CrossRef]

4. Ricke, S.C.; Richardson, K.; Dittoe, D.K. Formaldehydes in feed and their potential interaction with the poultry gastrointestinal tract microbial community-A review. Front. Vet. Sci. 2019, 6, 1-11. [CrossRef] [PubMed]

5. Li, M.; Lin, H.; Jing, Y.; Wang, J. Broad-host-range Salmonella bacteriophage STP4-a and its potential application evaluation in poultry industry. Poult. Sci. 2020, 99, 3643-3654. [CrossRef] [PubMed]

6. Swain, P.S.; Rao, S.B.N.; Rajendran, D.; Dominic, G.; Selvaraju, S. Nano zinc, an alternative to conventional zinc as animal feed supplement: A review. Anim. Nutr. 2016, 2, 134-141. [CrossRef]

7. Torres-Pitarch, A.; Manzanilla, E.G.; Gardiner, G.E.; O'Doherty, J.V.; Lawlor, P.G. Systematic review and meta-analysis of the effect of feed enzymes on growth and nutrient digestibility in grow-finisher pigs: Effect of enzyme type and cereal source. Anim. Feed Sci. Technol. 2019, 251, 153-165. [CrossRef]

8. Schneitz, C.; Hakkinen, M. The efficacy of a commercial competitive exclusion product on Campylobacter colonization in broiler chickens in a 5-week pilot-scale study. Poult. Sci. 2016, 95, 1125-1128. [CrossRef] [PubMed]

9. Celi, P.; Cowieson, A.J.; Fru-Nji, F.; Steinert, R.E.; Kluenter, A.M.; Verlhac, V. Gastrointestinal functionality in animal nutrition and health: New opportunities for sustainable animal production. Anim. Feed Sci. Technol. 2017, 234, 88-100. [CrossRef]

10. Broom, L.J.; Kogut, M.H. Inflammation: Friend or foe for animal production? Poult. Sci. 2018, 97, 510-514. [CrossRef]

11. Celi, P.; Verlhac, V.; Pérez Calvo, E.; Schmeisser, J.; Kluenter, A.M. Biomarkers of gastrointestinal functionality in animal nutrition and health. Anim. Feed Sci. Technol. 2019, 250, 9-31. [CrossRef]

12. Abd El-Hack, M.E.; El-Saadony, M.T.; Shafi, M.E.; Qattan, S.Y.A.; Batiha, G.E.; Khafaga, A.F.; Abdel-Moneim, A.M.E.; Alagawany, M. Probiotics in poultry feed: A comprehensive review. J. Anim. Physiol. Anim. Nutr. 2020, 104, 1835-1850. [CrossRef]

13. Kiarie, E.G.; Leung, H.; Akbari Moghaddam Kakhki, R.; Patterson, R.; Barta, J.R. Utility of feed enzymes and yeast derivatives in ameliorating deleterious effects of Coccidiosis on intestinal health and function in broiler chickens. Front. Vet. Sci. 2019, 6, 1-13. [CrossRef]

14. Kim, S.A.; Jang, M.J.; Kim, S.Y.; Yang, Y.; Pavlidis, H.O.; Ricke, S.C. Potential for prebiotics as feed additives to limit foodborne Campylobacter establishment in the poultry gastrointestinal tract. Front. Microbiol. 2019, 10, 1-12. [CrossRef]

15. Hoste, H.; Torres-Acosta, J.F.J.; Sandoval-Castro, C.A.; Mueller-Harvey, I.; Sotiraki, S.; Louvandini, H.; Thamsborg, S.M.; Terrill, T.H. Tannin containing legumes as a model for nutraceuticals against digestive parasites in livestock. Vet. Parasitol. 2015, 212, 5-17. [CrossRef] [PubMed]

16. Shang, Y.; Kumar, S.; Oakley, B.; Kim, W.K. Chicken gut microbiota: Importance and detection technology. Front. Vet. Sci. 2018, 5, 1-11. [CrossRef] [PubMed]

17. Ricke, S.C. Perspectives on the use of organic acids and short chain fatty acids as antimicrobials. Poult. Sci. 2003, 82, 632-639. [CrossRef]

18. Dibner, J.J.; Richards, J.D. Antibiotic growth promoters in agriculture: History and mode of action. Poult. Sci. 2005, 84, 634-643. [CrossRef]

19. Teirlynck, E.; Gussem, M.D.E.; Dewulf, J.; Haesebrouck, F.; Ducatelle, R.; Van Immerseel, F. Morphometric evaluation of "dysbacteriosis" in broilers. Avian Pathol. 2011, 40, 139-144. [CrossRef]

20. Vighi, G.; Marcucci, F.; Sensi, L.; Di Cara, G.; Frati, F. Allergy and the gastrointestinal system. Clin. Exp. Immunol. 2008, 153, 3-6. [CrossRef]

21. Yeoman, C.J.; White, B.A. Gastrointestinal tract microbiota and probiotics in production animals. Annu. Rev. Anim. Biosci. 2014, 2, 469-486. [CrossRef] [PubMed]

22. Windisch, W.; Schedle, K.; Plitzner, C.; Kroismayr, A. Use of phytogenic products as feed additives for swine and poultry. J. Anim. Sci. 2008, 86, E140-E148. [CrossRef]

23. Applegate, T.J.; Klose, V.; Steiner, T.; Ganner, A.; Schatzmayr, G. Probiotics and phytogenics for poultry: Myth or reality? J. Appl. Poult. Res. 2010, 19, 194-210. [CrossRef]

24. Gadde, U.; Kim, W.H.; Oh, S.T.; Lillehoj, H.S. Alternatives to antibiotics for maximizing growth performance and feed efficiency in poultry: A review. Anim. Health Res. Rev. 2017, 18, 26-45. [CrossRef]

25. Mohammadi Gheisar, M.; Kim, I.H. Phytobiotics in poultry and swine nutrition-A review. Ital. J. Anim. Sci. 2018, 17, 92-99. [CrossRef] 
26. Righi, F.; Pitino, R.; Manuelian, C.L.; Simoni, M.; Quarantelli, A.; De Marchi, M.; Tsiplakou, E. Plant feed additives as natural alternatives to the use of synthetic antioxidant vitamins on poultry performances, health, and oxidative status: A review of the literature in the last 20 years. Antioxidants 2021, 10, 659. [CrossRef] [PubMed]

27. Yitbarek, M.B. Phytogenics as feed additives in poultry production: A review. Int. J. Extensive Res. 2015, 3, 49-60.

28. Losa, R. The use of essential oils in animal nutrition. Feed Manuf. Mediterr. Reg. Improv. Saf. Feed Food 2001, 44, 39-44.

29. Miguel, M.G. Chemical variability of the leaf oil of 113 hybrids from Citrus clementina (Commun) $\times$ Citrus deliciosa (Willow leaf). Flavour Fragr. J. 2008, 23, 152-163. [CrossRef]

30. Bouhaddouda, N.; Aouadi, S.; Labiod, R. Evaluation of chemical composition and biological activities of essential oil and methanolic extract of Origanum vulgare L. ssp. glandulosum (DESF.) Ietswaart from Algeria. Int. J. Pharmacogn. Phytochem. Res. 2016, 8, 104-112.

31. Chouhan, S.; Sharma, K.; Guleria, S. Antimicrobial activity of some essential oils-Present status and future perspectives. Medicines 2017, 4, 58. [CrossRef]

32. Khattak, F.; Ronchi, A.; Castelli, P.; Sparks, N. Effects of natural blend of essential oil on growth performance, blood biochemistry, cecal morphology, and carcass quality of broiler chickens. Poult. Sci. 2014, 93, 132-137. [CrossRef]

33. Zhang, G.F.; Yang, Z.B.; Wang, Y.; Yang, W.R.; Jiang, S.Z.; Gai, G.S. Effects of ginger root (Zingiber officinale) processed to different particle sizes on growth performance, antioxidant status, and serum metabolites of broiler chickens. Poult. Sci. 2009, 88, 2159-2166. [CrossRef]

34. Ocak, N.; Erener, G.; Burak Ak, F.; Sungu, M.; Altop, A.; Ozmen, A. Performance of broilers fed diets supplemented with dry peppermint (Mentha piperita L.) or thyme (Thymus vulgaris L.) leaves as growth promoter source. Czech J. Anim. Sci. 2008, 53, 169-175. [CrossRef]

35. Bozkurt, M.; Küçükyılmaz, K.; Çatlı, A.; Çınar, M. Effect of dietary mannan oligosaccharide with or without oregano essential oil and hop extract supplementation on the performance and slaughter characteristics of male broilers. S. Afr. J. Anim. Sci. 2009, 39, 223-232. [CrossRef]

36. Jamroz, D.; Wiliczkiewicz, A.; Wertelecki, T.; Orda, J.; Skorupińska, J. Use of active substances of plant origin in chicken diets based on maize and locally grown cereals. Br. Poult. Sci. 2005, 46, 485-493. [CrossRef]

37. Amad, A.A.; Männer, K.; Wendler, K.R.; Neumann, K.; Zentek, J. Effects of a phytogenic feed additive on growth performance and ileal nutrient digestibility in broiler chickens. Poult. Sci. 2011, 90, 2811-2816. [CrossRef]

38. van der Aar, P.J.; Molist, F.; van der Klis, J.D. The central role of intestinal health on the effect of feed additives on feed intake in swine and poultry. Anim. Feed Sci. Technol. 2017, 233, 64-75. [CrossRef]

39. Paraskeuas, V.; Fegeros, K.; Palamidi, I.; Hunger, C.; Mountzouris, K.C. Growth performance, nutrient digestibility, antioxidant capacity, blood biochemical biomarkers and cytokines expression in broiler chickens fed different phytogenic levels. Anim. Nutr. 2017, 3, 114-120. [CrossRef]

40. Liu, S.D.; Song, M.H.; Yun, W.; Lee, J.H.; Lee, C.H.; Kwak, W.G.; Han, N.S.; Kim, H.B.; Cho, J.H. Effects of oral administration of different dosages of carvacrol essential oils on intestinal barrier function in broilers. J. Anim. Physiol. Anim. Nutr. 2018, 102, 1257-1265. [CrossRef]

41. Peng, Q.Y.; Li, J.D.; Li, Z.; Duan, Z.Y.; Wu, Y.P. Effects of dietary supplementation with oregano essential oil on growth performance, carcass traits and jejunal morphology in broiler chickens. Anim. Feed Sci. Technol. 2016, 214, 148-153. [CrossRef]

42. Reis, J.H.; Gebert, R.R.; Barreta, M.; Baldissera, M.D.; dos Santos, I.D.; Wagner, R.; Campigotto, G.; Jaguezeski, A.M.; Gris, A.; de Lima, J.L.F.; et al. Effects of phytogenic feed additive based on thymol, carvacrol and cinnamic aldehyde on body weight, blood parameters and environmental bacteria in broilers chickens. Microb. Pathog. 2018, 125, 168-176. [CrossRef] [PubMed]

43. Zhu, N.; Wang, J.; Yu, L.; Zhang, Q.; Chen, K.; Liu, B. Modulation of growth performance and intestinal microbiota in chickens fed plant extracts or virginiamycin. Front. Microbiol. 2019, 10, 1-16. [CrossRef]

44. Hafeez, A.; Männer, K.; Schieder, C.; Zentek, J. Effect of supplementation of phytogenic feed additives (powdered vs. encapsulated) on performance and nutrient digestibility in broiler chickens. Poult. Sci. 2016, 95, 622-629. [CrossRef] [PubMed]

45. Yu, C.; Wei, J.; Yang, C.; Yang, Z.; Yang, W.; Jiang, S. Effects of star anise (Illicium verum Hook.f.) essential oil on laying performance and antioxidant status of laying hens. Anim. Sci. J. 2018, 90, 3957-3966. [CrossRef]

46. Marume, U.; Mokagane, J.M.; Shole, C.O.; Hugo, A. Citrullus lanatus essential oils inclusion in diets elicit nutraceutical effects on egg production, egg quality, and physiological characteristics in layer hens. Poult. Sci. 2020, 99, 3038-3046. [CrossRef]

47. Puvača, N.; Lika, E.; Cocoli, S.; Kika, T.S.; Bursić, V.; Vuković, G.; Simin, M.T.; Petrovic, A.; Cara, M. Use of tea tree essential oil (Melaleuca alternifolia) in laying hen's nutrition on performance and egg fatty acid profile as a promising sustainable organic agricultural tool. Sustainability 2020, 12, 3420. [CrossRef]

48. Abdel-Wareth, A.A.A.; Lohakare, J.D. Productive performance, egg quality, nutrients digestibility, and physiological response of bovans brown hens fed various dietary inclusion levels of peppermint oil. Anim. Feed Sci. Technol. 2020, 145, 1-31. [CrossRef]

49. Karadağoğlu, Ö.; Şahin, T.; Ölmez, M.; Ahsan, U.; Özsoy, B.; Önk, K. Fatty acid composition of liver and breast meat of quails fed diets containing black cumin (Nigella sativa L.) and/or coriander (Coriandrum sativum L.) seeds as unsaturated fatty acid sources. Livest. Sci. 2019, 223, 164-171. [CrossRef]

50. Abd El-Hack, M.E.; Mahgoub, S.A.; Hussein, M.M.A.; Saadeldin, I.M. Improving growth performance and health status of meat-type quail by supplementing the diet with black cumin cold-pressed oil as a natural alternative for antibiotics. Environ. Sci. Pollut. Res. 2018, 25, 1157-1167. [CrossRef] 
51. Sokrollahi, B.; Sharifi, B. Effect of Nigella sativa seeds on growth performance, blood parameters, carcass quality and antibody production in Japanese quails. J. Livest. Sci. 2018, 9, 56-64.

52. Rahman, M.M.; Kim, S.J. Effects of dietary Nigella sativa seed supplementation on broiler productive performance, oxidative status and qualitative characteristics of thighs meat. Ital. J. Anim. Sci. 2016, 15, 241-247. [CrossRef]

53. Abou-Elkhair, R.; Selim, S.; Hussein, E. Effect of supplementing layer hen diet with phytogenic feed additives on laying performance, egg quality, egg lipid peroxidation and blood biochemical constituents. Anim. Nutr. 2018, 4, 394-400. [CrossRef]

54. Saleh, A.A.; Gawish, E.; Mahmoud, S.F.; Amber, K.; Awad, W.; Alzawqari, M.H.; Shukry, M.; Abdel-Moneim, A.M.E. Effect of natural and chemical colorant supplementation on performance, egg-quality characteristics, yolk fatty-acid profile, and blood constituents in laying hens. Sustainability 2021, 13, 4503. [CrossRef]

55. Kumar, P.; Patra, A.K. Beneficial uses of black cumin (Nigella sativa L.) seeds as a feed additive in poultry nutrition. Worlds. Poult. Sci. J. 2017, 73, 872-885. [CrossRef]

56. Hang, T.T.T.; Molee, W.; Khempaka, S.; Paraksa, N. Supplementation with curcuminoids and tuna oil influenced skin yellowness, carcass composition, oxidation status, and meat fatty acids of slow-growing chickens. Poult. Sci. 2018, 97, 901-909. [CrossRef] [PubMed]

57. Johannah, N.M.; Joseph, A.; Maliakel, B.; Krishnakumar, I.M. Dietary addition of a standardized extract of turmeric (TurmaFEED TM) improves growth performance and carcass quality of broilers. J. Anim. Sci. Technol. 2018, 60, 1-9. [CrossRef]

58. Oso, A.O.; Suganthi, R.U.; Reddy, G.B.M.; Malik, P.K.; Thirumalaisamy, G.; Awachat, V.B.; Selvaraju, S.; Arangasamy, A.; Bhatta, R. Effect of dietary supplementation with phytogenic blend on growth performance, apparent ileal digestibility of nutrients, intestinal morphology, and cecal microflora of broiler chickens. Poult. Sci. 2019, 98, 4755-4766. [CrossRef] [PubMed]

59. Shirani, V.; Jazi, V.; Toghyani, M.; Ashayerizadeh, A.; Sharifi, F.; Barekatain, R. Pulicaria gnaphalodes powder in broiler diets: Consequences for performance, gut health, antioxidant enzyme activity, and fatty acid profile. Poult. Sci. 2019, 98, $2577-2587$. [CrossRef] [PubMed]

60. Park, J.H.; Kim, I.H. Effects of dietary Achyranthes japonica extract supplementation on the growth performance, total tract digestibility, cecal microflora, excreta noxious gas emission, and meat quality of broiler chickens. Poult. Sci. 2019, 99, 463-470. [CrossRef] [PubMed]

61. Mohamed, S.H.; Attia, A.I.; Reda, F.M.; Abd El-Hack, M.E.; Ismail, I.E. Impacts of dietary supplementation of Boswellia serrata on growth, nutrients digestibility, immunity, antioxidant status, carcase traits and caecum microbiota of broilers. Ital. J. Anim. Sci. 2021, 20, 205-214. [CrossRef]

62. Herrero-Encinas, J.; Blanch, M.; Pastor, J.J.; Mereu, A.; Ipharraguerre, I.R.; Menoyo, D. Effects of a bioactive olive pomace extract from Olea europaea on growth performance, gut function, and intestinal microbiota in broiler chickens. Poult. Sci. 2020, 99, 2-10. [CrossRef]

63. Sharma, M.K.; Dinh, T.; Adhikari, P.A. Production performance, egg quality, and small intestine histomorphology of the laying hens supplemented with phytogenic feed additive. J. Appl. Poult. Res. 2020, 29, 362-371. [CrossRef]

64. Zhang, J.; Na, T.; Jin, Y.; Zhang, X.; Qu, H.; Zhang, Q. Thicker shell eggs with enriched N-3 polyunsaturated fatty acids and lower yolk cholesterol contents, as affected by dietary Nettle (Urtica cannabina) supplementation in laying hens. Animals 2020, 10, 1994. [CrossRef]

65. Ding, X.; Yu, Y.; Su, Z.; Zhang, K. Effects of essential oils on performance, egg quality, nutrient digestibility and yolk fatty acid profile in laying hens. Anim. Nutr. 2017, 3, 127-131. [CrossRef] [PubMed]

66. Uerlings, J.; Song, Z.G.; Hu, X.Y.; Wang, S.K.; Lin, H.; Buyse, J.; Everaert, N. Heat exposure affects jejunal tight junction remodeling independently of adenosine monophosphate-activated protein kinase in 9-day-old broiler chicks. Poult. Sci. 2018, 97, 3681-3690. [CrossRef] [PubMed]

67. Goo, D.; Kim, J.H.; Park, G.H.; Reyes, J.B.D.; Kil, D.Y. Effect of heat stress and stocking density on growth performance, breast meat quality, and intestinal barrier function in broiler chickens. Animals 2019, 9, 107. [CrossRef]

68. Akhavan-Salamat, H.; Ghasemi, H.A. Alleviation of chronic heat stress in broilers by dietary supplementation of betaine and turmeric rhizome powder: Dynamics of performance, leukocyte profile, humoral immunity, and antioxidant status. Trop. Anim. Health Prod. 2016, 48, 181-188. [CrossRef]

69. Song, Z.H.; Cheng, K.; Zheng, X.C.; Ahmad, H.; Zhang, L.L.; Wang, T. Effects of dietary supplementation with enzymatically treated Artemisia annua on growth performance, intestinal morphology, digestive enzyme activities, immunity, and antioxidant capacity of heat-stressed broilers. Poult. Sci. 2018, 97, 430-437. [CrossRef] [PubMed]

70. ur Rehman, Z.; Chand, N.; Khan, R.U. The effect of vitamin E, l-carnitine, and ginger on production traits, immune response, and antioxidant status in two broiler strains exposed to chronic heat stress. Environ. Sci. Pollut. Res. 2017, 24, 26851-26857. [CrossRef] [PubMed]

71. Zhang, J.F.; Bai, K.W.; Su, W.P.; Wang, A.A.; Zhang, L.L.; Huang, K.H.; Wang, T. Curcumin attenuates heat-stress-induced oxidant damage by simultaneous activation of GSH-related antioxidant enzymes and Nrf2-mediated phase II detoxifying enzyme systems in broiler chickens. Poult. Sci. 2018, 97, 1209-1219. [CrossRef]

72. Oke, O.E.; Oyelola, O.B.; Iyasere, O.S.; Njoku, C.P.; Oso, A.O.; Oso, O.M.; Fatoki, S.T.; Bankole, K.O.; Jimoh, I.O.; Sybill, N.I.; et al. In ovo injection of black cumin (Nigella sativa) extract on hatching and post hatch performance of thermally challenged broiler chickens during incubation. Poult. Sci. 2021, 100, 1-11. [CrossRef] [PubMed] 
73. Migliorini, M.J.; Boiago, M.M.; Stefani, L.M.; Zampar, A.; Roza, L.F.; Barreta, M.; Arno, A.; Robazza, W.S.; Giuriatti, J.; Galvão, A.C.; et al. Oregano essential oil in the diet of laying hens in winter reduces lipid peroxidation in yolks and increases shelf life in eggs. J. Therm. Biol. 2019, 85, 1-7. [CrossRef]

74. Abudabos, A.M.; Alyemni, A.H.; Dafalla, Y.M.; Khan, R.U. The effect of phytogenics on growth traits, blood biochemical and intestinal histology in broiler chickens exposed to Clostridium perfringens challenge. J. Appl. Anim. Res. 2018, 46, 691-695. [CrossRef]

75. Moharreri, M.; Vakili, R.; Oskoueian, E.; Rajabzadeh, G. Phytobiotic role of essential oil-loaded microcapsules in improving the health parameters in Clostridium perfringens -infected broiler chickens. Ital. J. Anim. Sci. 2021, 20, 2075-2085. [CrossRef]

76. Du, E.; Wang, W.; Gan, L.; Li, Z.; Guo, S.; Guo, Y. Effects of thymol and carvacrol supplementation on intestinal integrity and immune responses of broiler chickens challenged with Clostridium perfringens. J. Anim. Sci. Biotechnol. 2016, 7, 1-10. [CrossRef]

77. Moraes, P.O.; Cardinal, K.M.; Gouvêa, F.L.; Schroeder, B.; Ceron, M.S.; Lunedo, R.; Frazzon, A.P.G.; Frazzon, J.; Ribeiro, A.M.L. Comparison between a commercial blend of functional oils and monensin on the performance and microbiota of coccidiosischallenged broilers. Poult. Sci. 2019, 98, 5456-5464. [CrossRef]

78. Hussein, S.M.; M'Sadeq, S.A.; Beski, S.S.M.; Mahmood, A.L.; Frankel, T.L. Different combinations of peppermint, chamomile and a yeast prebiotic have different impacts on production and severity of intestinal and bursal abnormalities of broilers challenged with coccidiosis. Ital. J. Anim. Sci. 2021, 20, 1924-1934. [CrossRef]

79. Su, J.-L.; Shi, B.-L.; Zhang, P.-F.; Sun, D.-S.; Li, T.-Y.; Yan, S.-M. Effects of Yucca extract on feed efficiency, immune and antioxidative functions in broilers. Braz. Arch. Biol. Technol. 2016, 59, 1-8. [CrossRef]

80. Oelschlager, M.L.; Rasheed, M.S.A.; Smith, B.N.; Rincker, M.J.; Dilger, R.N. Effects of Yucca schidigera-derived saponin supplementation during a mixed Eimeria challenge in broilers. Poult. Sci. 2019, 98, 3212-3222. [CrossRef]

81. Yadav, S.; Teng, P.Y.; Souza dos Santos, T.; Gould, R.L.; Craig, S.W.; Lorraine Fuller, A.; Pazdro, R.; Kim, W.K. The effects of different doses of curcumin compound on growth performance, antioxidant status, and gut health of broiler chickens challenged with Eimeria species. Poult. Sci. 2020, 99, 5936-5945. [CrossRef]

82. Rajput, N.; Muhammad, N.; Yan, R.; Zhong, X.; Wang, T. Effect of dietary supplementation of curcumin on growth performance, intestinal morphology and nutrients utilization of broiler chicks. J. Poult. Sci. 2013, 50, 44-52. [CrossRef]

83. Liu, Z.; Wang, X.; Ou, S.; Arowolo, M.A.; Hou, D.X.; He, J. Effects of Achyranthes bidentata polysaccharides on intestinal morphology, immune response, and gut microbiome in yellow broiler chickens challenged with Escherichia coli K88. Polymers 2018, 10, 1233. [CrossRef] [PubMed]

84. Mohebodini, H.; Jazi, V.; Bakhshalinejad, R.; Shabani, A.; Ashayerizadeh, A. Effect of dietary resveratrol supplementation on growth performance, immune response, serum biochemical indices, cecal microflora, and intestinal morphology of broiler chickens challenged with Escherichia coli. Livest. Sci. 2019, 229, 13-21. [CrossRef]

85. Abudabos, A.M.; Alyemni, A.H.; Dafalla, Y.M.; Khan, R.U. The effect of phytogenic feed additives to substitute in-feed antibiotics on growth traits and blood biochemical parameters in broiler chicks challenged with Salmonella typhimurium. Environ. Sci. Pollut. Res. 2016, 23, 24151-24157. [CrossRef]

86. Pirgozliev, V.; Mansbridge, S.C.; Rose, S.P.; Lillehoj, H.S.; Bravo, D. Immune modulation, growth performance, and nutrient retention in broiler chickens fed a blend of phytogenic feed additives. Poult. Sci. 2019, 98, 3443-3449. [CrossRef] [PubMed]

87. Hassan, F.A.M.; Awad, A. Impact of thyme powder (Thymus vulgaris L.) supplementation on gene expression profiles of cytokines and economic efficiency of broiler diets. Environ. Sci. Pollut. Res. 2017, 24, 15816-15826. [CrossRef]

88. Mohiti-Asli, M.; Ghanaatparast-Rashti, M. Comparison of the effect of two phytogenic compounds on growth performance and immune response of broilers. J. Appl. Anim. Res. 2017, 45, 603-608. [CrossRef]

89. Dilawar, M.A.; Mun, H.S.; Rathnayake, D.; Yang, E.J.; Seo, Y.S.; Park, H.S.; Yang, C.J. Egg quality parameters, production performance and immunity of laying hens supplemented with plant extracts. Animals 2021, 11, 975. [CrossRef]

90. Kothari, D.; Oh, J.S.; Kim, J.H.; Lee, W.D.; Kim, S.K. Effect of dietary supplementation of fermented pine needle extract on productive performance, egg quality, and serum lipid parameters in laying hens. Animals 2021, 11, 1475. [CrossRef]

91. Moon, S.G.; Lee, S.K.; Do Lee, W.; Niu, K.M.; Hwang, W.U.; Oh, J.S.; Kothari, D.; Kim, S.K. Effect of dietary supplementation of a phytogenic blend containing Schisandra chinensis, Pinus densiflora, and Allium tuberosum on productivity, egg quality, and health parameters in laying hens. Anim. Biosci. 2021, 34, 285-294. [CrossRef]

92. Cimrin, T. Thyme (Thymbra spicata L.), rosemary (Rosmarinus officinalis L.) and vitamin E supplementation of laying hens. S. Afr. J. Anim. Sci. 2019, 49, 914-919. [CrossRef]

93. Saleh, A.A.; Kirrella, A.A.; Dawood, M.A.O.; Ebeid, T.A. Effect of dietary inclusion of cumin seed oil on the performance, egg quality, immune response and ovarian development in laying hens under high ambient temperature. J. Anim. Physiol. Anim. Nutr. 2019, 103, 1810-1817. [CrossRef]

94. Chen, Y.; Chen, H.; Li, W.; Miao, J.; Chen, N.; Shao, X.; Cao, Y. Polyphenols in Eucalyptus leaves improved the egg and meat qualities and protected against ethanol-induced oxidative damage in laying hens. J. Anim. Physiol. Anim. Nutr. 2018, 102, 214-223. [CrossRef] [PubMed]

95. Wang, X.C.; Wang, X.H.; Wang, J.; Wang, H.; Zhang, H.J.; Wu, S.G.; Qi, G.H. Dietary tea polyphenol supplementation improved egg production performance, albumen quality, and magnum morphology of hy-line brown hens during the late laying period1. $J$ Anim. Sci. 2018, 96, 225-235. [CrossRef] 
96. Jahanian, E.; Jahanian, R.; Rahmani, H.R.; Alikhani, M. Dietary supplementation of Echinacea purpurea powder improved performance, serum lipid profile, and yolk oxidative stability in laying hens. J. Appl. Anim. Res. 2017, 45, 45-51. [CrossRef]

97. Akbari, M.; Torki, M.; Kaviani, K. Single and combined effects of peppermint and thyme essential oils on productive performance, egg quality traits, and blood parameters of laying hens reared under cold stress condition $\left(6.8 \pm 3{ }^{\circ} \mathrm{C}\right)$. Int. J. Biometeorol. 2016, 60, 447-454. [CrossRef]

98. Kara, K.; Kocaoğlu Güçlü, B.; Baytok, E.; Şentürk, M. Effects of grape pomace supplementation to laying hen diet on performance, egg quality, egg lipid peroxidation and some biochemical parameters. J. Appl. Anim. Res. 2016, 44, 303-310. [CrossRef]

99. Vakili, R.; Majidzadeh Heravi, R. Performance and egg quality of laying hens fed diets supplemented with herbal extracts and flaxseed. Poult. Sci. J. 2016, 4, 107-116. [CrossRef]

100. da Rosa, G.; Dazuk, V.; Alba, D.F.; Galli, G.M.; Molosse, V.; Boiago, M.M.; Souza, C.F.; Abbad, L.B.; Baldissera, M.D.; Stefani, L.M.; et al. Curcumin addition in diet of laying hens under cold stress has antioxidant and antimicrobial effects and improves bird health and egg quality. J. Therm. Biol. 2020, 91, 1-10. [CrossRef] [PubMed]

101. Reis, J.H.; Gebert, R.R.; Barreta, M.; Boiago, M.M.; Souza, C.F.; Baldissera, M.D.; Santos, I.D.; Wagner, R.; Laporta, L.V.; Stefani, L.M.; et al. Addition of grape pomace flour in the diet on laying hens in heat stress: Impacts on health and performance as well as the fatty acid profile and total antioxidant capacity in the egg. J. Therm. Biol. 2019, 80, 141-149. [CrossRef] [PubMed]

102. Ao, X.; Kim, I.H. Effects of grape seed extract on performance, immunity, antioxidant capacity, and meat quality in Pekin ducks. Poult. Sci. 2020, 99, 2078-2086. [CrossRef]

103. Ding, X.; Wu, X.; Zhang, K.; Bai, S.; Wang, J.; Peng, H.W.; Xuan, Y.; Su, Z.; Zeng, Q. Dietary supplement of essential oil from oregano affects growth performance, nutrient utilization, intestinal morphology and antioxidant ability in Pekin ducks. J. Anim. Physiol. Anim. Nutr. 2020, 104, 1067-1074. [CrossRef] [PubMed]

104. Fathi, M.M.; Al-Homidan, I.; Ebeid, T.A.; Abou-Emera, O.K.; Mostafa, M.M. Dietary supplementation of Eucalyptus leaves enhances eggshell quality and immune response in two varieties of Japanese quails under tropical condition. Poult. Sci. 2020, 99, 879-885. [CrossRef]

105. Abouelezz, K.; Abou-Hadied, M.; Yuan, J.; Elokil, A.A.; Wang, G.; Wang, S.; Wang, J.; Bian, G. Nutritional impacts of dietary oregano and Enviva essential oils on the performance, gut microbiota and blood biochemicals of growing ducks. Animal 2019, 13, 2216-2222. [CrossRef]

106. Fernandez, M.E.; Kembro, J.M.; Ballesteros, M.L.; Caliva, J.M.; Marin, R.H.; Labaque, M.C. Dynamics of thymol dietary supplementation in quail (Coturnix japonica): Linking bioavailability, effects on egg yolk total fatty acids and performance traits. PLoS ONE 2019, 14, e0216623. [CrossRef]

107. Guo, L.; Hua, J.; Luan, Z.; Xue, P.; Zhou, S.; Wang, X.; Qin, N. Effects of the stems and leaves of Astragalus membranaceus on growth performance, immunological parameters, antioxidant status, and intestinal bacteria of quail. Anim. Sci. J. 2019, 90, 747-756. [CrossRef] [PubMed]

108. Mehri, M.; Sabaghi, V.; Bagherzadeh-Kasmani, F. Mentha piperita (peppermint) in growing Japanese quails diet: Performance, carcass attributes, morphology and microbial populations of intestine. Anim. Feed Sci. Technol. 2015, 207, 104-111. [CrossRef]

109. Hussein, E.O.S.; Ahmed, S.H.; Abudabos, A.M.; Suliman, G.M.; Abd El-Hack, M.E.; Swelum, A.A.; Alowaimer, A.N. Ameliorative effects of antibiotic-, probiotic-and phytobiotic-supplemented diets on the performance, intestinal health, carcass traits, and meat quality of clostridium perfringens-infected broilers. Animals 2020, 10, 669. [CrossRef]

110. Leskovec, J.; Levart, A.; Žgur, S.; Jordan, D.; Pirman, T.; Salobir, J.; Rezar, V. Effects of olive leaf and marigold extracts on the utilization of nutrients and on bone mineralization using two different oil sources in broilers. J. Poult. Sci. 2018, 55, 17-27. [CrossRef]

111. Pirgozliev, V.; Bravo, D.; Mirza, M.W.; Rose, S.P. Growth performance and endogenous losses of broilers fed wheat-based diets with and without essential oils and xylanase supplementation. Poult. Sci. 2015, 94, 1227-1232. [CrossRef] [PubMed]

112. Murugesan, G.R.; Syed, B.; Haldar, S.; Pender, C. Phytogenic feed additives as an alternative to antibiotic growth promoters in broiler chickens. Front. Vet. Sci. 2015, 2, 1-6. [CrossRef]

113. Gheisar, M.M.; Im, Y.W.; Lee, H.H.; Choi, Y.I.; Kim, I.H. Inclusion of phytogenic blends in different nutrient density diets of meat-type ducks. Poult. Sci. 2015, 94, 2952-2958. [CrossRef]

114. Reyer, H.; Zentek, J.; Männer, K.; Youssef, I.M.I.; Aumiller, T.; Weghuber, J.; Wimmers, K.; Mueller, A.S. Possible molecular mechanisms by which an essential oil blend from star anise, rosemary, thyme, and oregano and saponins increase the performance and ileal protein digestibility of growing broilers. J. Agric. Food Chem. 2017, 65, 6821-6830. [CrossRef]

115. Kers, J.G.; Velkers, F.C.; Fischer, E.A.J.; Hermes, G.D.A.; Stegeman, J.A.; Smidt, H. Host and environmental factors affecting the intestinal microbiota in chickens. Front. Microbiol. 2018, 9, 1-14. [CrossRef]

116. Mountzouris, K.C.; Paraskevas, V.; Tsirtsikos, P.; Palamidi, I.; Steiner, T.; Schatzmayr, G.; Fegeros, K. Assessment of a phytogenic feed additive effect on broiler growth performance, nutrient digestibility and caecal microflora composition. Anim. Feed Sci. Technol. 2011, 168, 223-231. [CrossRef]

117. Cross, D.E.; McDevitt, R.M.; Hillman, K.; Acamovic, T. The effect of herbs and their associated essential oils on performance, dietary digestibility and gut microflora in chickens from 7 to 28 days of age. Br. Poult. Sci. 2007, 48, 496-506. [CrossRef] [PubMed]

118. Kirkpinar, F.; Ünlü, H.B.; Özdemir, G. Effects of oregano and garlic essential oils on performance, carcase, organ and blood characteristics and intestinal microflora of broilers. Livest. Sci. 2011, 137, 219-225. [CrossRef] 
119. Hashemipour, H.; Kermanshahi, H.; Golian, A.; Raji, A. Effect of antibiotic alternatives on ileal microflora and intestinal histomorphology of broiler chickens fed wheat based diet. Iran. J. Appl. Anim. Sci. 2014, 4, 135-142.

120. Hashemipour, H.; Khaksar, V.; Rubio, L.A.; Veldkamp, T.; van Krimpen, M.M. Effect of feed supplementation with a thymol plus carvacrol mixture, in combination or not with an NSP-degrading enzyme, on productive and physiological parameters of broilers fed on wheat-based diets. Anim. Feed Sci. Technol. 2016, 211, 117-131. [CrossRef]

121. Park, J.H.; Kim, I.H. Effects of a protease and essential oils on growth performance, blood cell profiles, nutrient retention, ileal microbiota, excreta gas emission, and breast meat quality in broiler chicks. Poult. Sci. 2018, 97, 2854-2860. [CrossRef]

122. Chen, Y.; Ni, J.; Li, H. Effect of green tea and mulberry leaf powders on the gut microbiota of chicken. BMC Vet. Res. 2019, 15, 1-6. [CrossRef]

123. Perricone, V.; Comi, M.; Giromini, C.; Rebucci, R.; Agazzi, A.; Savoini, G.; Bontempo, V. Green tea and pomegranate extract administered during critical moments of the production cycle improves blood antiradical activity and alters cecal microbial ecology of broiler chickens. Animals 2020, 10, 785. [CrossRef] [PubMed]

124. Paraskeuas, V.V.; Mountzouris, K.C. Modulation of broiler gut microbiota and gene expression of Toll-like receptors and tight junction proteins by diet type and inclusion of phytogenics. Poult. Sci. 2019, 98, 2220-2230. [CrossRef]

125. Saeed, M.; Xu, Y.; Zhang, T.; Ren, Q.; Sun, C. 16S ribosomal RNA sequencing reveals a modulation of intestinal microbiome and immune response by dietary L-theanine supplementation in broiler chickens. Poult. Sci. 2019, 98, 842-854. [CrossRef]

126. Lan, Y.; Verstegen, M.W.A.; Tamminga, S.; Williams, B.A. The role of the commensal gut microbial community in broiler chickens. Worlds Poult. Sci. J. 2005, 61, 95-104. [CrossRef]

127. Lopetuso, L.R.; Scaldaferri, F.; Petito, V.; Gasbarrini, A. Commensal Clostridia: Leading players in the maintenance of gut homeostasis. Gut Pathog. 2013, 5, 1-8. [CrossRef]

128. Feng, J.; Lu, M.; Wang, J.; Zhang, H.; Qiu, K.; Qi, G.; Wu, S. Dietary oregano essential oil supplementation improves intestinal functions and alters gut microbiota in late-phase laying hens. J. Anim. Sci. Biotechnol. 2021, 12, 1-15. [CrossRef] [PubMed]

129. Placha, I.; Takacova, J.; Ryzner, M.; Cobanova, K.; Laukova, A.; Strompfova, V.; Venglovska, K.; Faix, S. Effect of thyme essential oil and selenium on intestine integrity and antioxidant status of broilers. Br. Poult. Sci. 2014, 55, 105-114. [CrossRef] [PubMed]

130. Du, E.; Gan, L.; Li, Z.; Wang, W.; Liu, D.; Guo, Y. In vitro antibacterial activity of thymol and carvacrol and their effects on broiler chickens challenged with Clostridium perfringens. J. Anim. Sci. Biotechnol. 2015, 6, 1-12. [CrossRef]

131. Yin, D.; Du, E.; Yuan, J.; Gao, J.; Wang, Y.L.; Aggrey, S.E.; Guo, Y. Supplemental thymol and carvacrol increases ileum Lactobacillus population and reduces effect of necrotic enteritis caused by Clostridium perfringes in chickens. Sci. Rep. 2017, 7, 1-11. [CrossRef]

132. Shipitsyna, E.; Roos, A.; Datcu, R.; Hallén, A.; Fredlund, H.; Jensen, J.S.; Engstrand, L.; Unemo, M. Composition of the vaginal microbiota in women of reproductive age-Sensitive and specific molecular diagnosis of bacterial vaginosis is possible? PLoS ONE 2013, 8, e60670. [CrossRef]

133. Gudiña, E.J.; Fernandes, E.C.; Teixeira, J.A.; Rodrigues, L.R. Antimicrobial and anti-adhesive activities of cell-bound biosurfactant from Lactobacillus agilis CCUG31450. RSC Adv. 2015, 5, 90960-90968. [CrossRef]

134. Khan, S.H. The use of green tea (Camellia sinensis) as a phytogenic substance in poultry diets. Onderstepoort J. Vet. Res. 2014, 81, 1-8. [CrossRef]

135. Zou, X.; Xiao, R.; Li, H.; Liu, T.; Liao, Y.; Wang, Y.; Wu, S.; Li, Z. Effect of a novel strain of Lactobacillus brevis M8 and tea polyphenol diets on performance, meat quality and intestinal microbiota in broilers. Ital. J. Anim. Sci. 2018, 17, 396-407. [CrossRef]

136. Muir, W.I.; Bryden, W.L.; Husband, A.J. Immunity, vaccination and the avian intestinal tract. Dev. Comp. Immunol. 2000, 24, 325-342. [CrossRef]

137. Mohiti-asli, M.; Ghanaatparast-rashti, M. Comparing the effects of a combined phytogenic feed additive with an individual essential oil of oregano on intestinal morphology and microflora in broilers. J. Appl. Anim. Res. 2018, 46, 184-189. [CrossRef]

138. Lillehoj, H.; Liu, Y.; Calsamiglia, S.; Miyakawa, M.E.F.; Chi, F.; Cravens, R.L.; Oh, S.; Gay, C.G. Phytochemicals as antibiotic alternatives to promote growth and enhance host health. Vet. Res. 2018, 49, 1-18. [CrossRef]

139. Lee, S.H.; Lillehoj, H.S.; Chun, H.K.; Tuo, W.; Park, H.J.; Cho, S.M.; Lee, Y.M.; Lillehoj, E.P. In vitro treatment of chicken peripheral blood lymphocytes, macrophages, and tumor cells with extracts of Korean medicinal plants. Nutr. Res. 2007, 27, 362-366. [CrossRef]

140. Lee, S.H.; Lillehoj, H.S.; Hong, Y.H.; Jang, S.I.; Lillehoj, E.P.; Ionescu, C.; Mazuranok, L.; Bravo, D. In vitro effects of plant and mushroom extracts on immunological function of chicken lymphocytes and macrophages. Br. Poult. Sci. 2010, 51, $213-221$. [CrossRef] [PubMed]

141. Kumar, S.; Ciraci, C.; Redmond, S.B.; Chuammitri, P.; Andreasen, C.B.; Palić, D.; Lamont, S.J. Immune response gene expression in spleens of diverse chicken lines fed dietary immunomodulators. Poult. Sci. 2011, 90, 1009-1013. [CrossRef] [PubMed]

142. Kim, D.K.; Lillehoj, H.S.; Lee, S.H.; Jang, S.I.; Bravo, D. High-throughput gene expression analysis of intestinal intraepithelial lymphocytes after oral feeding of carvacrol, cinnamaldehyde, or Capsicum oleoresin. Poult. Sci. 2010, 89, 68-81. [CrossRef] [PubMed]

143. Lillehoj, H.S. Recent Progress in Understanding Host Mucosal Response to Avian Coccidiosis and development of alternative strategies to mitigate the use of antibiotics in poultry production. Korean J. Poult. Sci. 2011, 38, 275-284. [CrossRef] 
144. Galli, G.M.; Gerbet, R.R.; Griss, L.G.; Fortuoso, B.F.; Petrolli, T.G.; Boiago, M.M.; Souza, C.F.; Baldissera, M.D.; Mesadri, J.; Wagner, R.; et al. Combination of herbal components (curcumin, carvacrol, thymol, cinnamaldehyde) in broiler chicken feed: Impacts on response parameters, performance, fatty acid profiles, meat quality and control of coccidia and bacteria. Microb. Pathog. 2020, 139, 1-11. [CrossRef]

145. El-Shall, N.A.; Shewita, R.S.; Abd El-Hack, M.E.; AlKahtane, A.; Alarifi, S.; Alkahtani, S.; Abdel-Daim, M.M.; Sedeik, M.E. Effect of essential oils on the immune response to some viral vaccines in broiler chickens, with special reference to Newcastle disease virus. Poult. Sci. 2020, 29, 2944-2954. [CrossRef] [PubMed]

146. Moraes, P.O.; Andretta, I.; Cardinal, K.M.; Ceron, M.; Vilella, L.; Borille, R.; Frazzon, A.P.; Frazzon, J.; Santin, E.; Ribeiro, A.M.L. Effect of functional oils on the immune response of broilers challenged with Eimeria spp. Animal 2019, 13, 2190-2198. [CrossRef]

147. Chowdhury, S.; Mandal, G.P.; Patra, A.K.; Kumar, P.; Samanta, I.; Pradhan, S.; Samanta, A.K. Different essential oils in diets of broiler chickens: 2 . Gut microbes and morphology, immune response, and some blood profile and antioxidant enzymes. Anim. Feed Sci. Technol. 2018, 236, 39-47. [CrossRef]

148. Lee, Y.; Lee, S.; Gadde, U.D.; Oh, S.; Lee, S.; Lillehoj, H.S. Dietary Allium hookeri reduces inflammatory response and increases expression of intestinal tight junction proteins in LPS-induced young broiler chicken. Res. Vet. Sci. 2017, 112, 149-155. [CrossRef]

149. El-GhAffAr GAlAl, A.A.A.; el-ArAby, I.E.-S.; Hassanin, O.; Omar, A.E.-S. Positive impact of oregano essential oil on growth performance, humoral immune responses and chicken interferon alpha signalling pathway in broilers. Adv. Anim. Vet. Sci. 2016, 4, 57-65. [CrossRef]

150. Lee, S.H.; Lillehoj, H.S.; Jang, S.I.; Lillehoj, E.P.; Min, W.; Bravo, D.M. Dietary supplementation of young broiler chickens with Capsicum and turmeric oleoresins increases resistance to necrotic enteritis. Br. J. Nutr. 2013, 110, 840-847. [CrossRef]

151. Ulmer-Franco, A.M.; Cherian, G.; Quezada, N.; Fasenko, G.M.; McMullen, L.M. Hatching egg and newly hatched chick yolk sac total IgY content at 3 broiler breeder flock ages. Poult. Sci. 2012, 91, 758-764. [CrossRef]

152. Sadeghi, G.H.; Karimi, A.; Padidar Jahromi, S.H.; Azizi, T.; Daneshmand, A. Effects of cinnamon, thyme and turmeric infusions on the performance and immune response in of 1- to 21-day-old male broilers. Braz. J. Poult. Sci. 2012, 14, 15-20. [CrossRef]

153. Toghyani, M.; Toghyani, M.; Gheisari, A.; Ghalamkari, G.; Eghbalsaied, S. Evaluation of cinnamon and garlic as antibiotic growth promoter substitutions on performance, immune responses, serum biochemical and haematological parameters in broiler chicks. Livest. Sci. 2011, 138, 167-173. [CrossRef]

154. Mehr, M.A.; Hassanabadi, A.; Moghaddam, H.N.; Kermanshahi, H. Supplementation of clove essential oils and probiotic on blood components, lymphoid organs and immune response in broiler chickens. Res. Opin. Anim. Vet. Sci. 2014, 4, $218-223$.

155. Hong, J.-C.; Steiner, T.; Aufy, A.; Lien, T.-F. Effects of supplemental essential oil on growth performance, lipid metabolites and immunity, intestinal characteristics, microbiota and carcass traits in broilers. Livest. Sci. 2012, 144, 253-262. [CrossRef]

156. Eladl, A.H.; Arafat, N.; El-Shafei, R.A.; Farag, V.M.; Saleh, R.M.; Awadin, W.F. Comparative immune response and pathogenicity of the H9N2 avian influenza virus after administration of Immulant ${ }^{\circledR}$, based on Echinacea and Nigella sativa, in stressed chickens. Comp. Immunol. Microbiol. Infect. Dis. 2019, 65, 165-175. [CrossRef] [PubMed]

157. Swamy, S.M.K.; Tan, B.K.H. Cytotoxic and immunopotentiating effects of ethanolic extract of Nigella sativa L. seeds. J. Ethnopharmacol. 2000, 70, 1-7. [CrossRef]

158. Ghasemi, H.A.; Kasani, N.; Taherpour, K. Effects of black cumin seed (Nigella sativa L.), a probiotic, a prebiotic and a synbiotic on growth performance, immune response and blood characteristics of male broilers. Livest. Sci. 2014, 164, 128-134. [CrossRef]

159. Toghyani, M.; Toghyani, M.; Shivazad, M.; Gheisari, A.; Bahadoran, R. Chromium supplementation can alleviate the negative effects of heat stress on growth performance, carcass traits, and meat lipid oxidation of broiler chicks without any adverse impacts on blood constituents. Biol. Trace Elem. Res. 2012, 146, 171-180. [CrossRef]

160. Yarmohammadi Barbarestani, S.; Jazi, V.; Mohebodini, H.; Ashayerizadeh, A.; Shabani, A.; Toghyani, M. Effects of dietary lavender essential oil on growth performance, intestinal function, and antioxidant status of broiler chickens. Livest. Sci. 2020, 233, 1-7. [CrossRef]

161. Paraskeuas, V.; Fegeros, K.; Palamidi, I.; Theodoropoulos, G.; Mountzouris, K.C. Phytogenic administration and reduction of dietary energy and protein levels affects growth performance, nutrient digestibility and antioxidant status of broilers. J. Poult. Sci. 2016, 53, 264-273. [CrossRef]

162. Mousavi, A.; Mahdavi, A.H.; Riasi, A.; Soltani-Ghombavani, M. Synergetic effects of essential oils mixture improved egg quality traits, oxidative stability and liver health indices in laying hens fed fish oil. Anim. Feed Sci. Technol. 2017, 234, 162-172. [CrossRef]

163. Torki, M.; Sedgh-Gooya, S.; Mohammadi, H. Effects of adding essential oils of rosemary, dill and chicory extract to diets on performance, egg quality and some blood parameters of laying hens subjected to heat stress. J. Appl. Anim. Res. 2018, 46, 1118-1126. [CrossRef]

164. Ciftci, M.; Simsek, U.G.; Yuce, A.; Yilmaz, O.; Dalkilic, B. Effects of dietary antibiotic and cinnamon oil supplementation on antioxidant enzyme activities, cholesterol levels and fatty acid compositions of serum and meat in broiler chickens. Acta Vet. Brno 2010, 79, 33-40. [CrossRef]

165. Hoffman-Pennesi, D.; Wu, C. The effect of thymol and thyme oil feed supplementation on growth performance, serum antioxidant levels, and cecal Salmonella population in broilers. J. Appl. Poult. Res. 2010, 19, 432-443. [CrossRef]

166. Polat, U.; Yesilbag, D.; Eren, M. Serum biochemical profile of broiler chickens fed diets containing rosemary and rosemary volatile oil. J. Biol. Environ. Sci. 2011, 5, 23-30. [CrossRef] 
167. Kamkar, A.; Javan, A.J.; Asadi, F.; Kamalinejad, M. The antioxidative effect of Iranian Mentha pulegium extracts and essential oil in sunflower oil. Food Chem. Toxicol. 2010, 48, 1796-1800. [CrossRef]

168. Kim, D.K.; Lillehoj, H.S.; Lee, S.H.; Jang, S.I.; Park, M.S.; Min, W.; Lillehoj, E.P.; Bravo, D. Immune effects of dietary anethole on Eimeria acervulina infection. Poult. Sci. 2013, 92, 2625-2634. [CrossRef]

169. Su, G.; Zhou, X.; Wang, Y.; Chen, D.; Chen, G.; Li, Y.; He, J. Effects of plant essential oil supplementation on growth performance, immune function and antioxidant activities in weaned pigs. Lipids Health Dis. 2018, 17, 1-10. [CrossRef]

170. Ahmed, S.T.; Islam, M.M.; Bostami, A.B.M.R.; Mun, H.S.; Kim, Y.J.; Yang, C.J. Meat composition, fatty acid profile and oxidative stability of meat from broilers supplemented with pomegranate (Punica granatum L.) by-products. Food Chem. 2015, 188, 481-488. [CrossRef]

171. Starčević, K.; Krstulović, L.; Brozić, D.; Maurić, M.; Stojević, Z.; Mikulec, Ž.; Bajić, M.; Mašek, T. Production performance, meat composition and oxidative susceptibility in broiler chicken fed with different phenolic compounds. J. Sci. Food Agric. 2014, 95, 1172-1178. [CrossRef] [PubMed]

172. Yang, C.; Chowdhury, M.A.K.; Hou, Y.; Gong, J. Phytogenic compounds as alternatives to in-feed antibiotics: Potentials and challenges in application. Pathogens 2015, 4, 137-156. [CrossRef] [PubMed]

173. Stevanović, Z.D.; Bošnjak-Neumüller, J.; Pajić-Lijaković, I.; Raj, J.; Vasiljević, M. Essential oils as feed additives-Future perspectives. Molecules 2018, 23, 1717. [CrossRef] [PubMed]

174. Beauchemin, K.A.; McGinn, S.M. Effects of various feed additives on the methane emissions from beef cattle. Int. Congr. Ser. 2006, 1293, 152-155. [CrossRef]

175. de Oliveira, E.F.; Paula, H.C.B.; de Paula, R.C.M. Alginate/cashew gum nanoparticles for essential oil encapsulation. Colloids Surf. B Biointerfaces 2014, 113, 146-151. [CrossRef]

176. Akbarzadeh, A.; Rezaei-Sadabady, R.; Davaran, S.; Joo, S.W.; Zarghami, N.; Hanifehpour, Y.; Samiei, M.; Kouhi, M.; Nejati-Koshki, K. Liposome: Classification, preparation, and applications. Nanoscale Res. Lett. 2013, 8, 1-9. [CrossRef]

177. Micciche, A.; Rothrock, M.J., Jr.; Yang, Y.; Ricke, S.C. Essential oils as an intervention strategy to reduce Campylobacter in poultry production: A review. Front. Microbiol. 2019, 10, 1-22. [CrossRef] [PubMed]

178. Bassolé, I.H.N.; Juliani, H.R. Essential oils in combination and their antimicrobial properties. Molecules 2012, $17,3989-4006$. [CrossRef]

179. Foskolos, A.; Ferret, A.; Siurana, A.; Castillejos, L.; Calsamiglia, S. Effects of capsicum and propyl-propane thiosulfonate on rumen fermentation, digestion, and milk production and composition in dairy cows. Animals 2020, 10, 859. [CrossRef]

180. Soltan, Y.A.; Natel, A.S.; Araujo, R.C.; Morsy, A.S.; Abdalla, A.L. Progressive adaptation of sheep to a microencapsulated blend of essential oils: Ruminal fermentation, methane emission, nutrient digestibility, and microbial protein synthesis. Anim. Feed Sci. Technol. 2018, 237, 8-18. [CrossRef]

181. Pham, V.H.; Kan, L.; Huang, J.; Geng, Y.; Zhen, W.; Guo, Y.; Abbas, W.; Wang, Z. Dietary encapsulated essential oils and organic acids mixture improves gut health in broiler chickens challenged with necrotic enteritis. J. Anim. Sci. Biotechnol. 2020, 11, 1-18 [CrossRef] [PubMed]

182. Stefanello, C.; Rosa, D.P.; Dalmoro, Y.K.; Segatto, A.L.; Vieira, M.S.; Moraes, M.L.; Santin, E. Protected blend of organic acids and essential oils improves growth performance, nutrient digestibility, and intestinal health of broiler chickens undergoing an intestinal challenge. Front. Vet. Sci. 2020, 6, 1-10. [CrossRef]

183. Nazzaro, F.; Fratianni, F.; De Martino, L.; Coppola, R.; De Feo, V. Effect of essential oils on pathogenic bacteria. Pharmaceuticals 2013, 6, 1451-1474. [CrossRef] [PubMed]

184. Mahmoud, B.S.M. The efficacy of grape seed extract, citric acid and lactic acid on the inactivation of Vibrio parahaemolyticus in shucked oysters. Food Control 2014, 41, 13-16. [CrossRef]

185. Feye, K.M.; Swaggerty, C.L.; Kogut, M.H.; Ricke, S.C.; Piva, A.; Grilli, E. The biological effects of microencapsulated organic acids and botanicals induces tissue-specific and dose-dependent changes to the Gallus gallus microbiota. BMC Microbiol. 2020, $20,1-15$. [CrossRef] [PubMed]

186. Stamilla, A.; Messina, A.; Sallemi, S.; Condorelli, L.; Antoci, F.; Puleio, R.; Loria, G.R.; Cascone, G.; Lanza, M. Effects of microencapsulated blends of organics acids (OA) and essential oils (EO) as a feed additive for broiler chicken. A focus on growth performance, gut morphology and microbiology. Animals 2020, 10, 442. [CrossRef] [PubMed]

187. Swaggerty, C.L.; He, H.; Genovese, K.J.; Callaway, T.R.; Kogut, M.H.; Piva, A.; Grilli, E. A microencapsulated feed additive containing organic acids, thymol, and vanillin increases in vitro functional activity of peripheral blood leukocytes from broiler chicks. Poult. Sci. 2020, 99, 3428-3436. [CrossRef]

188. Wang, H.; Liang, S.; Li, X.; Yang, X.; Long, F.; Yang, X. Effects of encapsulated essential oils and organic acids on laying performance, egg quality, intestinal morphology, barrier function, and microflora count of hens during the early laying period. Poult. Sci. 2019, 98, 6751-6760. [CrossRef]

189. Booth, I.R. Regulation of cytoplasmic pH in bacteria. Microbiol. Rev. 1985, 49, 359-378. [CrossRef] 